TRANSACTIONS OF THE

AMERICAN MATHEMATICAL SOCIETY

Volume 355, Number 1, Pages 407-432

S 0002-9947(02)03123-9

Article electronically published on September 6, 2002

\title{
PROPER ACTIONS ON COHOMOLOGY MANIFOLDS
}

\author{
HARALD BILLER
}

\begin{abstract}
Essential results about actions of compact Lie groups on connected manifolds are generalized to proper actions of arbitrary groups on connected cohomology manifolds. Slices are replaced by certain fiber bundle structures on orbit neighborhoods. The group dimension is shown to be effectively finite. The orbits of maximal dimension form a dense open connected subset. If some orbit has codimension at most 2 , then the group is effectively a Lie group.
\end{abstract}

\section{INTRODUCTION}

In the rich theory of continuous group actions on manifolds [2], 9, 14, 20, [28], 38], work has traditionally been focused on compact Lie groups. However, Palais [40] already drew attention to the larger class of proper actions, and the structure theory of general locally compact groups [26] is sufficiently well-developed to allow the generalization of many important results. This is the subject of the present paper. Already when actions of compact Lie groups are studied, it is natural to generalize manifolds to cohomology manifolds, which form a class of spaces with better inheritance properties.

Many results for which one aims concern properties of the set of stabilizers of an action. The price to be paid for considering arbitrary locally compact groups rather than Lie groups is that one does not get statements about the full stabilizers but about their identity components. However, this has the benefit that one does not need information about torsion in cohomology. Thus, unlike most of the classical theorems about actions of compact Lie groups, the present results hold not only for actions on cohomology manifolds with respect to integer coefficients but also for actions on cohomology manifolds over fields of characteristic 0 , and this is a strictly wider class.

An important preparatory result asserts that sheaf cohomological and covering dimension coincide for coset spaces of locally compact groups (1.1). Turning towards non-transitive proper actions, we show that each point has a neighborhood consisting of points for which the identity component of the stabilizer is, up to conjugation, contained in the stabilizer of the given point (2.8). For proper actions on completely regular spaces, Palais's Slice Theorem [40] is shown to have the following analog (3.8): each $n$-dimensional orbit has an invariant neighborhood that admits an equivariant fiber bundle projection onto a homogeneous $n$-manifold. A similar statement holds for infinite-dimensional orbits. The main result (4.11)

Received by the editors November 30, 2001, and, in revised form, April 6, 2002.

2000 Mathematics Subject Classification. Primary 57S10, 57S20; Secondary 57P05.

Key words and phrases. proper action; cohomology manifold; orbit structure; dimension estimate; slice; Hilbert-Smith conjecture. 
studies proper effective actions on a connected cohomology manifold over a field of characteristic 0. Among other things, we show that the set of points on highestdimensional orbits is open, connected, and dense since its complement has codimension at least 2. The group dimension is finite (in fact, a sharp upper bound in terms of the maximal orbit dimension is given). In the special case of actions of compact Lie groups on cohomology manifolds over $\mathbb{Z}$, these results are essentially due to Yang [51]. Corollary 4.13 asserts that the group is a Lie group if some orbit has codimension at most 2. This was first proved by Bredon [11 for actions of compact groups on manifolds. Raymond [42 later generalized it to cohomology manifolds over the integers under additional hypotheses which, in the light of the present treatment, seem unnaturally restrictive.

This paper exploits recent refinements of the general theory [26 in order to continue work by Yang [52], Bredon, Raymond, and Williams [17], and Raymond [42]. However, these authors emphasize certain surprising differences between actions of Lie and of non-Lie groups on cohomology manifolds over the integers. Their results, together with later work by Bredon [16, Section V.18], probably constitute the main step so far towards the proof of the Hilbert-Smith Conjecture, which states that a locally compact group that acts continuously and effectively on a connected manifold must be a Lie group. As mentioned above, this is true if the group is compact and some orbit has codimension at most 2 (Bredon [11]), even for cohomology manifolds (Raymond 42 and 4.13) and proper actions of non-compact groups (4.13). Without such a hypothesis, the conjecture has only been proved for actions by diffeomorphisms on differentiable manifolds (Montgomery [34] and Bochner and Montgomery [8]; cf. Upmeier [49, Section 11] for manifolds of infinite dimension) and, recently, for actions on Riemannian manifolds by Lipschitz homeomorphisms (Repovš and Ščepin [44]) or by quasi-conformal homeomorphisms (Martin [33]).

The results of this paper may be applied to topological incidence geometries in the sense of [19, Chapters 23 and 24] and [45]. In this theory, the customary hypotheses imply that the point set of an incidence geometry (such as a compact projective plane) is a cohomology manifold with respect to any coefficient domain [30, 32. Therefore, Theorem 4.11 and its Corollary 4.14 provide a uniform proof for the fact that the automorphism group of such a geometry, which carries a natural locally compact topology, has finite dimension. Previous proofs (e.g. [4], [48]) had to use separate lines of attack for each type of incidence axioms. Moreover, actions of compact Lie and non-Lie groups on such geometries used to be treated by rather different methods [4], [5. A unified approach will be possible in the light of the present results.

The second section of this paper develops parts of my Ph.D. thesis [4. This was written at the University of Stuttgart, Germany, under the supervision of Professor Markus Stroppel, whose advice and support I gratefully acknowledge.

\section{Dimension of COSET SPACES}

In this short section, we prove that covering and cohomological dimension coincide for coset spaces of locally compact groups, and we show when finite-dimensionality of such a coset space implies finite-dimensionality of the group. Note that in our terminology, the definition of (local) compactness includes the Hausdorff separation axiom. 
Finite-dimensional spaces are the main interest of this paper. Therefore, we need to provide a notion of the dimension of a topological space. Prominent dimension functions are covering dimension (dim) and small and large inductive dimension (ind and Ind; see, e.g., Engelking [23]). These functions take their values in $\mathbb{Z}_{\geq-1} \cup$ $\{\infty\}$. (Hofmann and Morris 25] have recently defined a cardinal-valued dimension function and studied its behavior on coset spaces of locally compact groups.) The three dimension functions, dim, ind, and Ind, do not agree in general, but they coincide for coset spaces of locally compact groups with respect to closed subgroups, as was proved by Pasynkov [41] (cf. Skljarenko [46. Theorem 9]). One can also use cohomology over some ring $R$ to define the cohomological dimension $\left(\operatorname{dim}_{R}\right)$ of a topological space. As we will see in Proposition 1.1 this coincides with the classical dimension functions on coset spaces of locally compact groups.

We will often use the following sum formula for the dimension of coset spaces of locally compact groups, which is due to Skljarenko [46. Theorem 10]. Let $G$ be a locally compact group, and let $H$ and $K$ be closed subgroups of $G$ such that $K \subseteq H$. Then

$$
\operatorname{dim} G / K=\operatorname{dim} G / H+\operatorname{dim} H / K .
$$

One of the most important facts about locally compact groups is that they can be approximated by Lie groups. To be precise, let $G$ be a locally compact group such that the quotient $G / G^{1}$ of $G$ by its identity component $G^{1}$ is compact. Then $G$ has arbitrarily small compact normal subgroups $N$ such that $G / N$ is a Lie group. If, moreover, the dimension of $G$ is finite, then every sufficiently small closed subgroup of $G$ is zero-dimensional. This was proved by Yamabe [50] and by Gluškov [24] Theorem 9]; see also Montgomery and Zippin [38, Chapter IV] and Kaplansky [29. II.10, Theorem 18]. Since being a Lie group is an extension property, if $N_{1}$ and $N_{2}$ are compact normal subgroups of $G$ such that $G / N_{1}$ and $G / N_{2}$ are Lie groups, then so is $G /\left(N_{1} \cap N_{2}\right)$ (cf. [24, 1.5]).

For the following proposition, recall the notion of the $\operatorname{dimension} \operatorname{dim}_{R} X$ of a topological space $X$ with respect to sheaf cohomology over a base ring $R$. For locally compact spaces $X$, this dimension is characterized by the fact that for any integer $n \geq-1$, the inequality $\operatorname{dim}_{R} X \leq n$ holds if and only if $H_{c}^{n+1}(U ; R)$ vanishes for all open subsets $U \subseteq X$, where $H_{c}^{*}(U ; R)$ denotes the cohomology of the constant sheaf with stalks $R$ on $U$ with respect to compact supports. For further information, the reader is referred to Bredon [16], Section II.16.

Proposition 1.1 (Cohomological and covering dimension). Let $H$ be a closed subgroup of a locally compact group $G$, and let $R$ be any ring. Then $\operatorname{dim}_{R} G / H=$ $\operatorname{dim} G / H$.

Proof. Since $G / H$ is paracompact (see, e.g., Bourbaki [10] Ch. III, §4, Prop. 13]), we have the inequality $\operatorname{dim}_{R} G / H \leq \operatorname{dim} G / H$ (Bredon [16, II.16.34]). On the other hand, if $\operatorname{dim} G / H \geq n$, then $G / H$ contains a homeomorphic image of $[0,1]^{n}$ by Theorem 9 of Skljarenko [46], so that $\operatorname{dim}_{R} G / H \geq n$ by [16, II.16.8 and II.16.28]. This yields the opposite inequality: $\operatorname{dim}_{R} G / H \geq \operatorname{dim} G / H$.

Theorem 1.2 (Transitive actions on finite-dimensional spaces). Let $G$ be a locally compact group, and let $H$ be a closed subgroup of $G$. Suppose that the coset space $G / H$ has finite dimension $n \in \mathbb{N}_{0}$ and that the action of $G$ on $G / H$ is effective. Let $G^{1} \subseteq G$ denote the connected component of the identity element, which is a closed normal subgroup of $G$. 
If $G \overline{G^{1} H}$ is compact, then the dimension of $G$ is finite.

If $G / H$ is connected and $H$ is compact, then $\operatorname{dim} G \leq\left(\begin{array}{c}n+1 \\ 2\end{array}\right)$.

Remark 1.3. (a) To see that the subgroup $G^{1} H$ need not be closed in $G$, take $G:=\mathbb{R} \times \mathbb{Z}_{p}$, where $\mathbb{Z}_{p}$ is the group of $p$-adic integers, and set $H:=\langle(1,1)\rangle$. Then $H$ is discrete, and $G^{1} H=\mathbb{R} \times \mathbb{Z}$ is a proper dense subgroup of $G$. In this example, the quotient $G / H$ is the $p$-adic solenoid. However, if $G$ is locally connected, then $G^{1}$ and $G^{1} H$ are open (hence closed) subgroups.

(b) Note that $G / \overline{G^{1} H}$ is homeomorphic to the space of connected components of $G / H$. To see this, define an equivalence relation on $G / H$ by setting $g H \sim g^{\prime} H$ if $g H$ and $g^{\prime} H$ belong to the same connected component, and equip the quotient space $X$ of $G / H$ by $\sim$ with the quotient topology. Then

$$
\begin{aligned}
\varphi: G / \overline{G^{1} H} & \longrightarrow X \\
g \overline{G^{1} H} & \longmapsto[g H]_{\sim}
\end{aligned}
$$

is a homeomorphism. Indeed, the subset $g \overline{G^{1} H} / H$ of $G / H$ is connected because it is the closure of the $G^{1}$-orbit of $g H$. This shows that $\varphi$ is well-defined. It is easy to see that it is a continuous surjection. Under the natural projection of $G / H$ onto $G / \overline{G^{1} H}$, the pre-image of an open-closed subset is open and closed. Therefore, such a pre-image is saturated under the equivalence relation $\sim$. Since the totally disconnected locally compact space $G \overline{G^{1} H}$ has a basis that consists of open-closed subsets, we conclude that $\varphi$ is open and injective.

(c) Let $K$ be a transitive permutation group on a set $S$, and equip both $K$ and $S$ with the respective discrete topology. Then there is an effective coset space of the semi-direct product $G:=\mathbb{T}^{S} \rtimes K$ that is homeomorphic to $\mathbb{S}_{1} \times S$ and hence a one-dimensional manifold, while the dimension of $G$ may be arbitrarily high and even infinite. For the case that the coset space is connected, the natural transitive action of the group $\mathrm{SO}_{n+1} \mathbb{R}$ on the $n$-sphere $\mathbb{S}_{n}$ shows that the upper bound for $\operatorname{dim} G$ is sharp.

Proof of Theorem 1.2. Since the totally disconnected locally compact group $G / G^{1}$ has small open subgroups, we may choose an open subgroup $G_{0}$ of $G$ such that $G_{0} / G^{1}$ is compact and hence $G_{0}$ can be approximated by Lie groups. We study the action of $G_{0}$ on $G / H$. The natural map from $G_{0}$ onto the orbit $G_{0} . g H$ is open, so that the action of $G_{0}$ on $G_{0} . g H$ is equivalent to the action on a coset space. Skljarenko [46. Theorem 5] has shown that $G_{0}$ is effectively finite-dimensional on $G_{0} . g H$. Since the orbits of $G_{0}$ in $G / H$ are all open, they are all closed. In particular, every connected component of $G / H$ is contained in a $G_{0}$-orbit. By Remark 1.3 , the space of connected components of $G / H$ is compact, which entails that the number of $G_{0}$-orbits in $G / H$ is finite. Therefore, the finiteness of $\operatorname{dim} G=\operatorname{dim} G_{0}$ is a consequence of the following fact: if $N_{1}, N_{2} \leq G_{0}$ are closed normal subgroups of finite codimension, then the codimension of $N_{1} \cap N_{2}$ in $G_{0}$ is finite. Indeed,

$$
\operatorname{dim} \frac{G_{0}}{N_{1} \cap N_{2}}=\operatorname{dim} \frac{G_{0}}{N_{1}}+\operatorname{dim} \frac{N_{1}}{N_{1} \cap N_{2}} \leq \operatorname{dim} \frac{G_{0}}{N_{1}}+\operatorname{dim} \frac{G_{0}}{N_{2}}
$$

by Skljarenko's sum formula (1) and because $N_{1} /\left(N_{1} \cap N_{2}\right)$ admits a continuous injection into $G_{0} / N_{2}$ (use cohomological dimension and see Bredon [16, IV.8.4]).

Now suppose that $G / H$ is connected and that $H$ is compact. Then the action of $G_{0}$ on $G / H$ is transitive, and it is equivalent to the action of $G_{0}$ on a coset space because $G_{0}$ is open in $G$. Therefore, we may assume that $G_{0}=G$. 
Let $N$ be a zero-dimensional compact normal subgroup of $G$ such that the quotient $G / N$ is a Lie group. The coset space $H N / H$ is zero-dimensional because it

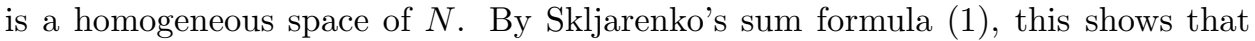
$\operatorname{dim} G / H N=\operatorname{dim} G / H$. It also implies that $(H N)^{1}=H^{1}$, whence the transitive action of the Lie group $G / N$ on $G / H N$ is almost effective (i.e., its kernel is totally disconnected). Therefore, we may even assume that $G$ is a Lie group. The action of the compact Lie group $H$ on the connected manifold $G / H$ is not transitive. Hence all orbits of $H$ in $G / H$ have dimension at most $n-1$ by domain invariance (see, for instance, Bredon [15, IV.19.9]). A result due to Montgomery and Zippin [38, 6.3.1, Theorem 2] yields that $\operatorname{dim} H=\operatorname{dim} H^{1} \leq\left(\begin{array}{l}n \\ 2\end{array}\right)$, whence $\operatorname{dim} G=\operatorname{dim} G / H+\operatorname{dim} H \leq\left(\begin{array}{c}n+1 \\ 2\end{array}\right)$.

\section{NEIGHBORING ORBITS OF PROPER ACTIONS}

From now on, our attention is focused on proper actions, which generalize actions of compact groups. In the remaining sections, we treat actions on general topological spaces, on completely regular spaces, and, finally, on (cohomology) manifolds.

In proper actions, the definition of which will soon be recalled, stabilizers depend semi-continuously on points (2.2), and a similar statement holds for the kernels of the restricted actions on single orbits (2.4). Stronger results hold for proper actions of locally compact groups. Using a small generalization of a classical theorem of Palais about proper actions of Lie groups (2.7), the main result of this section (2.8) studies proper actions of general locally compact groups and describes the behavior of stabilizers and kernels in a neighborhood of a finite-dimensional orbit. Two corollaries conclude the section.

Let us turn to the definitions. Since it appears to be textbook tradition to consider proper actions on non-Hausdorff spaces as well, we call a topological space quasi-compact if every open covering has a finite subcover, so that a (locally) compact space is a (locally) quasi-compact Hausdorff space. A continuous map $f: X \rightarrow Y$ is called proper if it is closed and all points of $Y$ have quasi-compact pre-images. This implies that the pre-image of every quasi-compact subset of $Y$ is quasi-compact; if $Y$ is locally compact, then the reverse implication holds as well. For example, if a quasi-compact group $N$ acts continuously on a topological space $X$, then the orbit projection $X \rightarrow X / N$ is a proper open map.

A continuous action of a topological group $G$ on a topological space $X$ is called proper if the map $(g, x) \mapsto(x, g \cdot x): G \times X \rightarrow X \times X$ is proper. Proper maps and actions are described by Bourbaki [10, Ch. I $\S 10$, Ch. III $\S 4]$; see also tom Dieck [20] I.3]. We recall the most important elementary properties of a proper action of $G$ on $X$. The orbit space $X / G$ is always a Hausdorff space, and if $G$ satisfies the Hausdorff property, then so does $X$. All stabilizers are quasi-compact, and for each $x \in X$, the map $g \mapsto g . x: G \rightarrow X$ is proper, so that the natural bijection of $G / G_{x}$ onto $G . x$ is a homeomorphism onto a closed subspace of $X$. A subgroup $H \leq G$ is quasi-compact if and only if the action of $G$ on the coset space $G / H$ is proper. A continuous action of a locally compact group $G$ on a Hausdorff space $X$ is proper if and only if for all $x, y \in X$, there are neighborhoods $V_{x}$ of $x$ and $V_{y}$ of $y$ such that $\left\{g \in G \mid g . V_{x} \cap V_{y} \neq \emptyset\right\}$ is relatively compact in $G$. In particular, every continuous action of a compact group on a Hausdorff space is proper. A continuous action of a Hausdorff group $G$ on a locally compact space $X$ is proper if and only if for each compact subset $K \subseteq X$, the closed subset $\{g \in G \mid g . K \cap K \neq \emptyset\}$ of $G$ is compact. 
Note that a Hausdorff group that acts properly on a nonempty (locally) compact space is (locally) compact.

In the investigation of neighboring orbits, the following characterization of proper actions is most useful: a continuous action is proper if and only if its stabilizers are quasi-compact, and the set of group elements that map one point to another depends semi-continuously on that pair of points. Writing $\mathcal{U}(S)$ for the neighborhood filter of a subset $S$ of a topological space, we formulate this characterization precisely:

Proposition 2.1. Let $G$ act continuously on a topological space $X$. Then the action is proper if and only if all stabilizers are quasi-compact and

$$
\begin{aligned}
\forall x, y \in X, U \in \mathcal{U}(\{g \in G \mid g \cdot x=y\}) \exists V_{x} \in \mathcal{U}(x), V_{y} \in \mathcal{U}(y): & \\
& \left\{g \in G \mid g \cdot V_{x} \cap V_{y} \neq \emptyset\right\} \subseteq U .
\end{aligned}
$$

Proof. The map $\theta: G \times X \rightarrow X \times X,(g, x) \mapsto(x, g \cdot x)$ has quasi-compact fibers if and only if all stabilizers are quasi-compact. Suppose that this condition is satisfied. As we will show, condition (2) is then equivalent to

$$
\forall x, y \in X, U \in \mathcal{U}\left(\theta^{-1}(x, y)\right) \exists V \in \mathcal{U}((x, y)): \theta^{-1}(V) \subseteq U,
$$

and this holds if and only if $\theta$ is a closed map.

Indeed, assume (2), choose $x, y \in X$, and let $U$ be a neighborhood of

$$
\theta^{-1}(x, y)=\{g \in G \mid g \cdot x=y\} \times\{x\} .
$$

Since this fiber is quasi-compact, there are neighborhoods $U^{\prime}$ of $\{g \in G \mid g \cdot x=y\}$ and $V_{x}^{\prime}$ of $x$ such that $U^{\prime} \times V_{x}^{\prime} \subseteq U$. According to (2), there are neighborhoods $V_{x}$ of $x$ and $V_{y}$ of $y$ such that $\left\{g \in G \mid g \cdot V_{x} \cap V_{y} \neq \emptyset\right\} \subseteq U^{\prime}$. Then $\theta^{-1}\left(\left(V_{x} \cap V_{x}^{\prime}\right) \times V_{y}\right) \subseteq$ $U^{\prime} \times V_{x}^{\prime} \subseteq U$, which shows that (3) holds. Conversely, if $x, y \in X$ and $U$ is a neighborhood of $\{g \in G \mid g \cdot x=y\}$, then (3) implies that there are neighborhoods $V_{x}$ of $x$ and $V_{y}$ such that $\theta^{-1}\left(V_{x} \times V_{y}\right) \subseteq U \times X$, which entails (2).

Now assume (3), let $A$ be a closed subset of $G \times X$, and choose $(x, y) \in(X \times X) \backslash$ $\theta(A)$. Then there is a neighborhood $V$ of $(x, y)$ such that $\theta^{-1}(V) \subseteq(G \times X) \backslash A$. Hence $V \cap \theta(A)=\emptyset$, and $\theta$ is a closed map. Conversely, assume that $\theta$ is closed, pick $x, y \in X$, and let $U$ be an open neighborhood of $\theta^{-1}(x, y)$. Then $V:=$ $(X \times X) \backslash \theta((G \times X) \backslash U)$ is an open neighborhood of $(x, y)$ with $\theta^{-1}(V) \subseteq U$, whence (3) holds.

Setting $x=y$ in (2), we obtain semi-continuous dependence of stabilizers on points:

Corollary 2.2 (Close points have close stabilizers). Let $G$ act properly on a space $X$, choose a point $x \in X$, and let $U$ be a neighborhood of the stabilizer $G_{x}$ of $x$. Then $x$ has a neighborhood $V$ such that $U$ contains the stabilizers of all points in $V$ :

$$
\exists V \in \mathcal{U}(x) \forall y \in V: G_{y} \subseteq U .
$$

From now on, we will always assume the Hausdorff separation property. In this setting, there is an analogous result for kernels of the actions on neighboring orbits. In order to deduce this from Corollary 2.2 we need a lemma about uniform spaces. For the sake of simplicity, it will be formulated as a statement about topological groups. 
Lemma 2.3. Let $G$ be a Hausdorff group, let $\mathcal{K}$ be a set of compact subsets of $G$, and let $U$ be a neighborhood of $\cap \mathcal{K}$ in $G$. Then there is a neighborhood $V$ of the identity in $G$ and a finite subset $\left\{K_{1}, \ldots, K_{n}\right\} \subseteq \mathcal{K}$ such that

$$
\bigcap_{i=1}^{n} K_{i} V \subseteq U
$$

Proof. We may assume that $U$ is open in $G$. By compactness, there is a finite subset $\left\{K_{1}, \ldots, K_{n}\right\} \subseteq \mathcal{K}$ such that $\bigcap_{i=1}^{n} K_{i} \subseteq U$. Let $C:=K_{1} \times \cdots \times K_{n} \subseteq G^{n}$, and set

$$
D:=\left\{(x, x, \ldots, x) \in G^{n} \mid x \in G \backslash U\right\} .
$$

Then $C$ is compact, the subset $D$ is closed, and $C \cap D=\emptyset$, whence there is a neighborhood $V$ of 1 in $G$ such that the neighborhood $K_{1} V \times \cdots \times K_{n} V$ of $C$ does not meet $D$. This implies that $\bigcap_{i=1}^{n} K_{i} V$ is contained in $U$.

Lemma 2.4 (Close orbits have close kernels). Let $G$ be a Hausdorff group acting properly on a space $X$, choose a point $x \in X$, and let $U$ be a neighborhood of the kernel $G_{[G . x]}$ of the action on the orbit G.x. Then $x$ has a neighborhood $V$ such that $U$ contains all kernels that correspond to orbits of points in $V$ :

$$
\exists V \in \mathcal{U}(x) \forall y \in V: G_{[G . y]} \subseteq U .
$$

Proof. Applying Lemma 2.3 to $\mathcal{K}:=\left\{g G_{x} g^{-1} \mid g \in G\right\}$, we find a neighborhood $W$ of $1 \in G$ and a finite subset $F \subseteq G$ such that

$$
\bigcap_{g \in F} g G_{x} g^{-1} W \subseteq U \text {. }
$$

Set $W^{\prime}:=\bigcap_{g \in F} g^{-1} W g$. Corollary 2.2 yields a neighborhood $V$ of $x \in X$ such that $G_{y} \subseteq G_{x} W^{\prime}$ holds for each $y \in V$. This entails

$$
G_{[G . y]} \subseteq \bigcap_{g \in F} g G_{y} g^{-1} \subseteq \bigcap_{g \in F} g G_{x} W^{\prime} g^{-1} \subseteq \bigcap_{g \in F} g G_{x} g^{-1} W \subseteq U .
$$

We record another consequence of Lemma 2.3 .

Corollary 2.5 (Comparison of group topologies). Let $G$ be a Hausdorff group acting properly and effectively on a topological space $X$. Then the given topology on $G$ coincides with the topologies on $G$ induced from the compact-open topology and from the product topology on $X^{X}$.

Proof. The compact-open topology is finer than the product topology. The given topology is finer than the compact-open topology on $G$ because continuity of the action implies that the natural injective map from $G$ into $X^{X}$ is continuous with respect to the given topology on $G$ and the compact-open topology on $X^{X}$ (Dugundji [21, XII.3.1]). It remains to see that the product topology on $G$ is finer than the given topology. To achieve this, choose $g_{0} \in G$, and let $U$ be a neighborhood of $g_{0}$ in $G$ with respect to the given topology. For each $x \in X$, set $K_{x}:=\left\{g \in G \mid g . x=g_{0} . x\right\}$. The intersection of these compact sets is $\left\{g_{0}\right\}$ because the action is effective. By Lemma 2.3, we can choose an identity neighborhood $V$ in $G$ and a finite subset $F \subseteq X$ such that $\bigcap_{x \in F} K_{x} V \subseteq U$. For each $x \in F$, Proposition 2.1 yields an open neighborhood $W_{x}$ of $g_{0} . x$ in $X$ such that $g . x \in W_{x}$ implies $g \in K_{x} V$. The set $\left\{g \in G \mid \forall x \in F: g . x \in W_{x}\right\}$ contains $g_{0}$ and is contained in $U$, and it is open with respect to the product topology. 
Remark 2.6. (a) Since we followed the tradition and admitted non-Hausdorff groups in the first results about proper actions, it may be interesting to note that already the preceding three results break down without the Hausdorff hypothesis. Indeed, topologize the symmetric group $G:=S_{3}$ in such a way that $G$ and its three-element subgroup are the open identity neighborhoods, let $H \leq G$ be a subgroup of order 2 , and let $X$ be the quotient space of $G / H \times[0,1]$ obtained by identifying, for each $t \in] 0,1]$, the set $G / H \times\{t\}$ to a point. Then the natural action of $G$ on $X$ is proper by Proposition 2.1, but it does not satisfy the conclusion of Lemma 2.4 and the compact-open topology on $G$ is indiscrete.

(b) For the sake of completeness and comparison with results given in the monograph by Montgomery and Zippin [38], we note that analogs of Corollary 2.2 and Lemma 2.4 hold for the identity components of stabilizers and kernels. This is due to the following topological fact: if $H$ is a locally compact (hence closed) subgroup of a Hausdorff group $G$ and $U$ is a neighborhood of the identity component $H^{1}$ in $G$, then there is a neighborhood $V$ of $H$ such that for every subgroup $K$ of $G$ contained in $V$, the identity component $K^{1}$ lies within $U$.

Indeed, we may suppose that $U$ is open in $G$. Since the totally disconnected locally compact group $H / H^{1}$ has small open subgroups, there is an open (and closed) subgroup $L$ of $H$ that lies within $U$. Under the natural map of $G$ onto the coset space $G / L$, the image of $L$ is a point and the image of $H \backslash L$ is a closed set. Since the topology of $G / L$ is regular, these two images have disjoint neighborhoods. Thus we obtain disjoint open neighborhoods $V_{1}$ of $L$ and $V_{2}$ of $H \backslash L$ in $G$. Set $V:=\left(U \cap V_{1}\right) \cup V_{2}$. Then $V$ is an open neighborhood of $H$ that has the desired property.

Stronger results hold for proper actions of locally compact groups if we suppose that the group is a Lie group (Proposition 2.7) or if we restrict our attention to identity components (Theorem 2.8).

Proposition 2.7 (Stabilizers in proper actions of Lie groups). Let a Lie group $G$ act properly on a space $X$, choose a point $x \in X$, and let $U$ be a neighborhood of the identity element in $G$. Then there is a neighborhood $V$ of $x$ such that all stabilizers of points in $V$ are conjugate to subgroups of the stabilizer $G_{x}$ by elements of $U$ :

$$
\exists V \in \mathcal{U}(x) \forall y \in V \exists g \in U: g G_{y} g^{-1} \subseteq G_{x} .
$$

Proof. Choose a point $x \in X$. Since the stabilizer $G_{x}$ is compact, a result by Montgomery and Zippin [37] shows that $G_{x}$ has a neighborhood $W$ such that every subgroup of $G$ contained in $W$ is conjugate to a subgroup of $G_{x}$ by an element of $U$. (Palais [40, 4.2] later re-proved this as a corollary to his Slice Theorem.) By Corollary 2.2] there is a neighborhood $V$ of $x$ such that all stabilizers of points in $V$ are contained in $W$.

Under the additional condition that $X$ is completely regular, the preceding result is due to Palais [40, 2.3, Corollary 2]. If we had not recorded the present easy generalization, we would have to assume complete regularity for the remainder of this section, which would be sufficient for the purposes of this paper.

Theorem 2.8 (Stabilizers in proper actions of locally compact groups). Let $G$ be a locally compact group acting properly on a space $X$, and suppose that the orbit $G . x$ of $x \in X$ has finite dimension. Let $U$ be a neighborhood of the identity element in $G$. 
Then there is a neighborhood $V$ of $x$ such that all identity components of stabilizers of points in $V$ are conjugate to subgroups of the stabilizer $G_{x}$ by elements of $U$ :

$$
\exists V \in \mathcal{U}(x) \forall y \in V \exists g \in U: g\left(G_{y}\right)^{1} g^{-1} \subseteq G_{x} .
$$

In particular, the relations $\operatorname{dim} G . y \geq \operatorname{dim} G . x$ and $\left(G_{[G . y]}\right)^{1} \subseteq G_{[G . x]}$ hold for each point $y \in V$, where $G_{[G . x]}$ denotes the kernel of the action of $G$ on $G . x$. Moreover, if $\operatorname{dim} G . y=\operatorname{dim} G . x$, then $g\left(G_{y}\right)^{1} g^{-1}=\left(G_{x}\right)^{1}$ and $\left(G_{[G . y]}\right)^{1}=\left(G_{[G . x]}\right)^{1}$.

Proof. Since $\left(G_{y}\right)^{1}=\left(\left(G^{1}\right)_{y}\right)^{1}$, the main assertion can be proved within the identity component. Write $K:=\left(G^{1}\right)_{\left[G^{1} . x\right]}$ for the kernel of the action of $G^{1}$ on the orbit $G^{1} . x$. Skljarenko's sum formula (1) entails that $\operatorname{dim} G^{1} \cdot x=\operatorname{dim} G$.x. Hence Theorem 1.2 implies that the group $G^{1} / K$ has finite dimension. Therefore, the compact subgroup $K$ is contained in a compact normal subgroup $N$ of $G^{1}$ such that the quotient $G^{1} / N$ is a Lie group and $N / K$ is zero-dimensional. In other words, the identity component $N^{1}$ is contained in $K$ and hence in $\left(G^{1}\right)_{x}$. This means that the orbit $N . x$ is zero-dimensional.

The natural action of $G^{1}$ on the orbit space $X / N$ factors through an action of the Lie group $G^{1} / N$. Both actions are proper, and we will apply Proposition 2.7 to the latter. For $y \in X$, the stabilizer of $N . y \in X / N$ in $G^{1} / N$ is $\left(G^{1}\right)_{N . y} / N$ (and $\left.\left(G^{1}\right)_{N . y}=\left(G^{1}\right)_{y} N\right)$. Moreover, two subgroups of $G^{1} / N$ are conjugate if and only if their pre-images in $G^{1}$ are conjugate. Therefore, Proposition 2.7 yields a neighborhood $V$ of $x$ such that for all $y \in V$, there is a $g \in U \cap G^{1}$ such that $g\left(G^{1}\right)_{N . y} g^{-1} \subseteq\left(G^{1}\right)_{N . x}$. Since $N . x$ is zero-dimensional, the identity component $\left(\left(G^{1}\right)_{N . x}\right)^{1}$ fixes $x$. Hence

$$
g\left(G_{y}\right)^{1} g^{-1}=g\left(\left(G^{1}\right)_{y}\right)^{1} g^{-1} \subseteq g\left(\left(G^{1}\right)_{N . y}\right)^{1} g^{-1} \subseteq\left(\left(G^{1}\right)_{N . x}\right)^{1} \subseteq G_{x} .
$$

Skljarenko's sum formula (1) yields

$$
\operatorname{dim} G . y=\operatorname{dim} \frac{G}{g G_{y} g^{-1}}=\operatorname{dim} \frac{G}{g\left(G_{y}\right)^{1} g^{-1}} \geq \operatorname{dim} \frac{G}{G_{x}}=\operatorname{dim} G . x .
$$

Moreover, we find that

$$
\begin{aligned}
\left(G_{[G . y]}\right)^{1} & =\left(\bigcap_{h \in G} h G_{y} h^{-1}\right)^{1}=\left(\bigcap_{h \in G} h\left(G_{y}\right)^{1} h^{-1}\right)^{1} \\
& =\left(\bigcap_{h \in G} h g\left(G_{y}\right)^{1} g^{-1} h^{-1}\right)^{1} \\
& \subseteq\left(\bigcap_{h \in G} h\left(G_{x}\right)^{1} h^{-1}\right)^{1}=\left(\bigcap_{h \in G} h G_{x} h^{-1}\right)^{1}=\left(G_{[G . x]}\right)^{1} .
\end{aligned}
$$

If $\operatorname{dim} G \cdot y=\operatorname{dim} G$.x, then $g\left(G_{y}\right)^{1} g^{-1}=\left(G_{x}\right)^{1}$ by Skljarenko's sum formula (1), whence also $\left(G_{[G . y]}\right)^{1}=\left(G_{[G . x]}\right)^{1}$.

In actions of compact non-Lie groups, the full stabilizer can "jump up", and even its identity component can do the same when the orbit dimension is infinite. Montgomery and Zippin [36, p. 786] describe an example which proves the first half of this assertion, and an example for the second half can be constructed along the same lines (see [4, 2.1.16] for details).

We finish this section with some immediate consequences of Theorem 2.8 . 
Corollary 2.9 (Orbits of maximal dimension). Let $G$ be a locally compact group acting properly on a space $X$. Suppose that

$$
k:=\max \{\operatorname{dim} G \cdot x \mid x \in X\}<\infty,
$$

and let $Y \subseteq X$ be the set of points on $k$-dimensional orbits. Then $Y$ is an open subset of $X$. Moreover, every point $x \in Y$ has an open neighborhood $V$ such that the identity components of all kernels of actions on orbits that meet $V$ coincide, and the identity components of all stabilizers of points in $V$ are conjugate.

If $G / G^{1}$ is compact, then the effective quotient $\left.G\right|_{G . V}$ of $G$ with respect to the action on the open set $G . V$ is finite-dimensional. If $G$ is connected, then $\left.\operatorname{dim} G\right|_{G . V} \leq\left(\begin{array}{c}k+1 \\ 2\end{array}\right)$.

Proof. Suppose that $x \in Y$, set $U:=G$, and choose an open neighborhood $V$ of $x$ as in Theorem 2.8. Then $V \subseteq Y$. Moreover, for all $y \in V$, we have

$$
\left(G_{[G . y]}\right)^{1}=\left(G_{[G . x]}\right)^{1} \quad \text { and } \quad \exists g \in G: g\left(G_{y}\right)^{1} g^{-1}=\left(G_{x}\right)^{1} .
$$

The last claim follows from Theorem 1.2 on transitive actions.

Corollary 2.10 (Uniform orbit dimension). Let $G$ be a locally compact group acting properly and effectively on a connected space $X$, and suppose that all orbits have the same finite dimension $k$. Then the identity components of all stabilizers are conjugate, the action of $G$ on every single orbit is almost effective, and the dimension of $G$ is at most $\left(\begin{array}{c}k+1 \\ 2\end{array}\right)$.

Proof. Skljarenko's sum formula (II) yields the equations $\operatorname{dim} G^{1}=\operatorname{dim} G$ and $\operatorname{dim} G^{1} \cdot x=\operatorname{dim} G . x$ for every point $x \in X$. Since the conclusions of the corollary follow for $G$ once they are proved for the identity component $G^{1}$, we may assume that $G$ is connected.

Choose a point $x \in X$, and set

$$
Y:=\left\{y \in X \mid \exists g \in G: g\left(G_{y}\right)^{1} g^{-1}=\left(G_{x}\right)^{1}\right\} .
$$

Then $Y$ is an open subset of $X$ by Corollary 2.9, If $z$ is a point on the topological boundary of $Y$, then $z \in Y$ by the same corollary. Thus $Y$ is closed as well, and $Y=X$ by connectedness. Hence

$$
\forall y \in X \exists g \in G: g\left(G_{y}\right)^{1} g^{-1}=\left(G_{x}\right)^{1} .
$$

As in the proof of Theorem 2.8, we find that the identity component of the kernel of the action on G.x satisfies

$$
\left(G_{[G . x]}\right)^{1}=\left(\bigcap_{g \in G} g\left(G_{x}\right)^{1} g^{-1}\right)^{1} .
$$

Since this is the same for every orbit and $G$ acts effectively, we conclude that $\left(G_{[G . x]}\right)^{1}=1$, so that the action of $G$ on $G \cdot x$ is almost effective. Using the assumption that $G$ is connected, we conclude from Skljarenko's sum formula (1) and Theorem 1.2 that

$$
\operatorname{dim} G=\operatorname{dim} \frac{G}{G_{[G . x]}} \leq\left(\begin{array}{c}
k+1 \\
2
\end{array}\right) .
$$


For actions of compact connected groups $G$, the last part of Corollary 2.10 was first stated by Montgomery and Zippin [36, Theorem 11] (cf. [38, 6.2.5]). However, there seems to be an essential gap in their proof. They choose $x \in X$ such that $\operatorname{dim} G /\left(G_{[G . x]}\right)^{1}$ is as large as possible. This choice allows them to infer that

$$
Y:=\left\{y \in X \mid\left(G_{[G . y]}\right)^{1}=\left(G_{[G . x]}\right)^{1}\right\}
$$

is an open subset of $X$. They claim without any further argument that $Y$ coincides with the fixed point set of $\left(G_{[G . x]}\right)^{1}$. This would imply that $Y$ is closed, so that $Y=X$ by connectedness, which finishes the proof.

In spite of this lapse, it should be emphasized that Section 6.2 of the classic book by Montgomery and Zippin [38] has provided much inspiration for the present material and, in particular, for Theorem 2.8

\section{SLICES}

A fundamental result for proper actions of Lie groups on completely regular spaces, due to Palais [40], asserts that every orbit is an equivariant neighborhood retract. In the main result of this section (3.8), we drop the Lie hypothesis from this situation. Then every orbit has an invariant neighborhood that admits an equivariant fiber bundle projection onto a homogeneous manifold; if the orbit dimension is finite, then that manifold may be chosen of the same dimension, and its dimension can be arbitrarily high if the orbit dimension is infinite. We also observe (3.2) that such an orbit neighborhood is equivariantly homeomorphic to a twisted product.

Let $G$ be a Hausdorff group, and let $H \leq G$ be a closed subgroup that acts on a Hausdorff space $A$. Then $H$ acts freely on $G \times A$ by $h .(g, a):=\left(g h^{-1}, h . a\right)$. The twisted product $G \times{ }_{H} A$ is defined as the orbit space of this action, and we write $[g, a]$ for the $H$-orbit of $(g, a) \in G \times A$. The group $G$ acts on $G \times{ }_{H} A$ by $g \cdot\left[g^{\prime}, a\right]:=\left[g g^{\prime}, a\right]$, and we have the $G$-equivariant projection

$$
[g, a] \longmapsto g H: G \times_{H} A \longrightarrow G / H .
$$

Our interest in twisted products lies in the fact that for a locally compact group $G$, every $G$-equivariant map onto a coset space of $G$ is of this form (Proposition 3.2). Thus twisted products provide a concrete description of such equivariant maps. For their elementary properties, see Bredon [14, I.6] or tom Dieck [20, I.4]. We add the following observations.

Lemma 3.1. Let $G$ be a Hausdorff group, and let $H$ be a closed subgroup that acts on a Hausdorff space $A$. Then the following assertions hold:

(a) The free action of $H$ on $G \times A$ given by $h .(g, a):=\left(g h^{-1}, h . a\right)$ is proper. In particular, the twisted product $G \times_{H} A$ is a Hausdorff space.

(b) Let $C$ be a compact subset of $G$. Then the restriction of the orbit projection pr: $G \times A \rightarrow G \times \times_{H} A$ to $C \times A$ is a proper map. In particular, the map $a \mapsto[1, a]: A \rightarrow G \times_{H} A$ is a closed embedding.

Proof. (a) This follows easily from the characterization of proper actions in Proposition 2.1] Indeed, if $h \cdot\left(g_{1}, a_{1}\right)=\left(g_{2}, a_{2}\right)$ and $U$ is a neighborhood of $h$ in $H$, then $h=g_{2}{ }^{-1} g_{1}$, and we may choose neighborhoods $V_{1}$ of $g_{1}$ and $V_{2}$ of $g_{2}$ such that $V_{2}{ }^{-1} V_{1} \cap H \subseteq U$. Then every element $h^{\prime} \in H$ for which $h^{\prime} .\left(V_{1} \times A\right)$ meets $V_{2} \times A$ is contained in $U$. If the $H$-orbits of $\left(g_{1}, a_{1}\right)$ and of $\left(g_{2}, a_{2}\right)$ do not meet, then there are two cases. If $g_{1} H \neq g_{2} H$, there are neighborhoods $V_{1}$ of $g_{1}$ and $V_{2}$ of $g_{2}$ such that $V_{1} H$ and $V_{2} H$ are disjoint, and then $h .\left(V_{1} \times A\right)$ and $V_{2} \times A$ are disjoint for 
every element $h \in H$. If $g_{1} H=g_{2} H$, then $g_{2}{ }^{-1} g_{1} \in H$ and $\left(g_{2}{ }^{-1} g_{1}\right) \cdot a_{1} \neq a_{2}$. We may choose neighborhoods $V_{1}$ of $g_{1}, V_{2}$ of $g_{2}, W_{1}$ of $a_{1}$, and $W_{2}$ of $a_{2}$ such that $\left(V_{2}{ }^{-1} V_{1} \cap H\right) . W_{1}$ is disjoint from $W_{2}$. Then $h .\left(V_{1} \times W_{1}\right)$ and $V_{2} \times W_{2}$ are disjoint for every element $h \in H$.

(b) The fiber through $(g, a) \in C \times A$ of the restriction $\left.\operatorname{pr}\right|_{C \times A}$ is the set $\left\{\left(g h, h^{-1} . a\right) \mid h \in H \cap g^{-1} C\right\}$, which is compact. The saturation of a closed subset $F \subseteq C \times A$ under the action of $H$ on $G \times A$ is the image of the closed set $H \times F$ under the concatenation

$$
\begin{array}{rlrl}
H \times C \times A & \stackrel{\cong}{\rightrightarrows} H \times C \times A \hookrightarrow G \times C \times A & \cong & \cong \\
(h, c, a) & \mapsto(h, c, h . a) \quad(g, c, a) & \mapsto\left(c g^{-1}, c, a\right)
\end{array}
$$

where the second map is the inclusion, the fourth map is the product projection, and the first and third maps are the indicated homeomorphisms. This concatenation is proper because it is a composition of proper maps. Hence $\left.\mathrm{pr}\right|_{C \times A}$ is a closed map.

Proposition 3.2. Let $G$ be a locally compact group acting on a Hausdorff space $X$, let $H \leq G$ be a closed subgroup, and suppose that there is a continuous $G$-equivariant map $\varphi: X \rightarrow G / H$. Set $A:=\varphi^{-1}(H)$. Then the following statements hold:

(a) The map $\psi: G \times_{H} A \rightarrow X,[g, a] \mapsto g$.a is a $G$-equivariant homeomorphism.

(b) Assume that the natural projection $\mathrm{pr}: G \rightarrow G / H$ admits continuous local cross sections. (By a result of Mostert's [39, Theorem 8], this condition is satisfied if the dimension of $G / H$ is finite; cf. Skljarenko [46. Theorem 13].) Then the map $\varphi$ is the projection in a locally trivial fiber bundle with fiber A.

Proof. (a) Define a continuous map $\tilde{\psi}: G \times A \rightarrow X,(g, a) \mapsto g$.a. This map is surjective because $\varphi(x)=g H$ implies $g^{-1} \cdot x \in A$. For $g, g^{\prime} \in G$ and $a, a^{\prime} \in A$, we have $\tilde{\psi}(g, a)=\tilde{\psi}\left(g^{\prime}, a^{\prime}\right)$ if and only if $a=g^{-1} g^{\prime} \cdot a^{\prime}$, which holds if and only if $g^{-1} g^{\prime} \in H$ and $(g, a)=g^{-1} g^{\prime}$. $\left(g^{\prime}, a^{\prime}\right)$, i.e., $[g, a]=\left[g^{\prime}, a^{\prime}\right]$. Hence $\tilde{\psi}$ factors through $\psi$, and $\psi$ is a $G$-equivariant continuous bijection.

Choose a point $[g, a] \in G \times{ }_{H} A$. Let $U$ be a compact neighborhood of $g$ in $G$, and let $V$ be the image of $U \times A$ under the natural projection of $G \times A$ onto $G \times{ }_{H} A$. Then $\psi(V)$ is the closed neighborhood $\tilde{\psi}(U \times A)=\varphi^{-1}(U H / H)$ of $\psi([g, a])$. The restriction of $\tilde{\psi}$ to $U \times A$ is a proper map (see Bourbaki [10, Ch. III, §4, Prop. 1]). Therefore, the map from $V$ onto $\psi(V)$ induced by $\psi$ is closed and hence a homeomorphism. We conclude that $\psi$ is a homeomorphism.

(b) Existence of local cross sections for pr: $G \rightarrow G / H$ means that there is an open subset $U \subseteq G / H$ and a continuous map $\sigma: U \rightarrow G$ such that $\operatorname{pr} \circ \sigma=\operatorname{id}_{U}$. (For examples of compact pairs $(G, H)$ without local cross sections, see Mostert [39] p. 59] or Skljarenko [46, p. 72].) Assertion (b) follows easily. Indeed, if $g \in G$, then one checks that

$$
\begin{aligned}
\chi_{g}: g U \times A & \longrightarrow \varphi^{-1}(g U) \\
(u, a) & \longmapsto g \sigma\left(g^{-1} u\right) . a
\end{aligned}
$$

is a continuous map with continuous inverse

$$
\begin{aligned}
\chi_{g}^{\prime}: \varphi^{-1}(g U) & \longrightarrow g U \times A \\
x & \longmapsto\left(\varphi(x), \sigma\left(g^{-1} \varphi(x)\right)^{-1} g^{-1} \cdot x\right),
\end{aligned}
$$


and $\varphi \circ \chi_{g}: g U \times A \rightarrow g U \subseteq G / H$ is the projection onto the first factor. Hence $\chi_{g}$ is a topological bundle chart over $g U$.

Definition 3.3. Let $G$ be a locally compact group acting on a Hausdorff space $X$, and let $H$ be a closed subgroup of $G$. Following Palais [40, 2.1.1], we call a nonempty subset $S \subseteq X$ an $H$-slice in $X$ for the action of $G$ if any one of the following conditions, which are equivalent by Proposition 3.2, is satisfied:

(i) There is a $G$-invariant open subset $U \subseteq X$ that admits a $G$-equivariant map $\varphi: U \rightarrow G / H$ such that $S=\varphi^{-1}(H)$. (Note that $U=G . S$, and also $\varphi$ is determined uniquely by $S$ because $\varphi(g . s)=g H$.)

(ii) There is an $H$-space $A$ and a $G$-equivariant open embedding

$$
\psi: G \times_{H} A \rightarrow X
$$

such that $\psi([1, A])=S$.

(iii) The subset $S$ is invariant under $H$, the subset $G . S \subseteq X$ is open, and

$$
\begin{aligned}
\chi: G \times_{H} S & \longrightarrow G . S \\
{[g, s] } & \longmapsto g . s
\end{aligned}
$$

is a homeomorphism.

Note that if $G \rightarrow G / H$ has local cross sections (e.g. if the dimension of $G / H$ is finite), then $\varphi: G . S \rightarrow G / H$ is a locally trivial fiber bundle with fiber $S$. Another fact which will be used is that an open $H$-invariant subset of an $H$-slice is an $H$-slice.

Let $G$ be a locally compact group acting properly on a completely regular space $X$ such that the orbit space $X / G$ is paracompact. Assume that $G / G^{1}$ is compact, and let $K$ be a maximal compact subgroup of $G$. Abels [1] has constructed a global $K$ slice $S$ in $X$ for the action of $G$, i.e. a $G$-equivariant homeomorphism from $G \times{ }_{K} S$ onto $X$. As $G / K$ is homeomorphic to some euclidean space, this implies that $X$ is a trivial fiber bundle over $G / K$, i.e., homeomorphic to $G / K \times S$. The purposes of the present paper, on the other hand, require the construction of (non-global) $H$-slices for which $H$ is as small as possible. It turns out that we do not need any assumptions on $X / G$ and $G / G^{1}$.

Lemma 3.4. Let $G$ be a locally compact group such that $G / G^{1}$ is compact, let $H \leq G$ be a closed subgroup, and let $\mathcal{N}$ be the set of compact normal subgroups of $G$ such that the factor group is a Lie group. If $N_{1} \in \mathcal{N}$ is such that $\operatorname{dim} G / H N_{1}<$ $\operatorname{dim} G / H$, then there is $N_{2} \in \mathcal{N}$ such that $N_{2} \subseteq N_{1}$ and $\operatorname{dim} G / H N_{2}>\operatorname{dim} G / H N_{1}$.

In particular, if the dimension of $G / H$ is finite, then there is $N \in \mathcal{N}$ such that $\operatorname{dim} G / H N=\operatorname{dim} G / H$. If the dimension of $G / H$ is infinite, then $N \in \mathcal{N}$ can be chosen such that $\operatorname{dim} G / H N$ is arbitrarily high.

Proof. By Skljarenko's sum formula (1), the dimension of $N_{1} H / H$ is strictly positive. Therefore, the stabilizer $N_{1} \cap H$ of $H$ under the action of $N_{1}$ on this space does not contain the identity component $N_{1}{ }^{1}$, so that there is a neighborhood $U$ of $H$ in $G / H$ that does not contain $N_{1}{ }^{1} H / H$. Choose $N \in \mathcal{N}$ such that $N H / H \subseteq U$. Then $N_{2}:=N \cap N_{1}$ is an element of $\mathcal{N}$ (Gluškov [24, 1.5]). The identity component $N_{1}{ }^{1}$ is not contained in $N_{2} H$, so that it acts nontrivially on $N_{1} H / N_{2} H$. Hence the dimension of this space is strictly positive, and the assertion follows from Skljarenko's sum formula (1). 
Lemma 3.5. If $H$ is a compact subgroup of a totally disconnected locally compact group $G$, then every neighborhood of $H$ contains an open subgroup $G_{0}$ of $G$, such that $H \in G_{0}$.

Proof. Since $H$ is compact, it suffices to consider neighborhoods of the form $U H$, where $U$ is an identity neighborhood in $G$. Since $G / H$ is totally disconnected, we may assume that $U H$ is compact and open in $G$. Then there is an identity neighborhood $V$ in $G$ such that $V \subseteq U$ and $U H V \subseteq U H$, whence $U H V H \subseteq U H$. By induction, we find that $(V H)^{n} \subseteq U H$ for each $n \in \mathbb{N}$. Therefore, the subgroup of $G$ that is generated by $V H \cap(V H)^{-1}$ is contained in $U H$, and it is open and contains $H$.

Lemma 3.6. Let $G$ be a locally compact group acting properly on a space $X$. Choose $x \in X$, let $G_{0} \leq G$ be an open subgroup with $G_{x} \subseteq G_{0}$, and let $H \leq G_{0}$ be a closed subgroup. Suppose that $S$ is an $H$-slice in $X$ for $G_{0}$ with $x \in S$. Then there is an $H$-slice $S^{\prime}$ in $X$ for $G$ such that $x \in S^{\prime} \subseteq S$.

Proof. Proposition 2.1 allows us to choose an open neighborhood $V$ of $x$ in $X$ such that $\{g \in G \mid g . V \cap V \neq \emptyset\} \subseteq G_{0}$. Since we can replace $V$ with $H . V$, we may assume that $V$ is $H$-invariant. Then $S^{\prime}:=S \cap V$ is also $H$-invariant, and it is open in $S$ and thus an $H$-slice for $G_{0}$. To show that it also is an $H$-slice for $G$, observe that $G . S^{\prime}=G .\left(G_{0} \cdot S^{\prime}\right)$ is open in $X$, so that it remains to prove that

$$
\begin{aligned}
\varphi: G . S^{\prime} & \longrightarrow G / H \\
g . s & \longmapsto g H
\end{aligned}
$$

is a well-defined continuous map. The basic reason for this is that $G \cdot S^{\prime}$ is a disjoint union of $G$-translates of $G_{0} . S^{\prime}$. To be precise, suppose that $g . s=g^{\prime} . s^{\prime}$, where $g, g^{\prime} \in G$ and $s, s^{\prime} \in S^{\prime}$. Then $s=g^{-1} g^{\prime} . s^{\prime} \in V \cap g^{-1} g^{\prime} V$, which implies that $g^{-1} g^{\prime} \in G_{0}$. Hence $g^{-1} g^{\prime} \in H$ because $S^{\prime}$ is an $H$-slice for $G_{0}$. For the same reason, the restriction $\left.\varphi\right|_{G_{0} . S^{\prime}}$ is continuous, which entails continuity of $\varphi$ at g.s (with $g \in G$ and $s \in S^{\prime}$ ). Indeed, if $x^{\prime}$ is an element of the open neighborhood $g G_{0} . S^{\prime}$ of $g . s$, then $g^{-1} . x^{\prime} \in G_{0} . S^{\prime}$ and $\varphi\left(x^{\prime}\right)=g \varphi\left(g^{-1} \cdot x^{\prime}\right)$ depends continuously on $x^{\prime}$.

Proposition 3.7. Let $G$ be a locally compact group acting properly on a completely regular space $X$. If $x \in X$ and $N$ is a compact normal subgroup of $G$ such that $G / N$ is a Lie group, then $x$ is contained in a $G_{x} N$-slice for the action of $G$ on $X$.

Proof. For $N=1$, i.e., for proper actions of Lie groups, this is the main result of Palais's seminal paper [40, 2.3.3]. (Note that his definition of a proper action implies the customary definition that we use, which in turn implies his definition of a "Cartan $G$-space".) The present situation is easily reduced to Palais's Theorem. Let pr: $X \rightarrow X / N$ denote the natural projection. Since this is a proper map, the action of $G / N$ on $X / N$ is proper. Moreover, Palais [40, 1.2.8] proved that $X / N$ is completely regular. (The action of $N$ on $X$ is proper in Palais's stronger sense because $N$ is compact.) Therefore, there is a $G / N$-invariant open neighborhood $U$ of $\operatorname{pr}(x)$ in $X / N$ that admits a $G$-equivariant map

$$
\varphi: U \longrightarrow G / G_{x} N \approx \frac{G / N}{G_{x} N / N}
$$


such that $\varphi(\operatorname{pr}(x))=G_{x} N$. Set $U^{\prime}:=\operatorname{pr}^{-1}(U)$. Then $U^{\prime}$ is a $G$-invariant open neighborhood of $x$ in $X$, and $\left.\varphi \circ \mathrm{pr}\right|_{U^{\prime}}$ is a $G$-equivariant map of $U^{\prime}$ onto $G / G_{x} N$ that sends $x$ to $G_{x} N$.

Theorem 3.8 (Existence of slices). Let $G$ be a locally compact group acting properly on a completely regular space $X$, and choose $x \in X$. Then there is a convergent filter basis $\mathcal{N}$ that consists of compact subgroups of $G$ normalized by $G_{x}$ such that for every $N \in \mathcal{N}$, the coset space $G / G_{x} N$ is a manifold and $x$ is contained in a $G_{x} N$-slice for the action of $G$ on $X$. In particular, some neighborhood of the orbit $G . x$ is a locally trivial fiber bundle over the manifold $G / G_{x} N$.

The dimension of $G . x$ is infinite if and only if $N \in \mathcal{N}$ may be chosen such that the dimension of $G / G_{x} N$ is arbitrarily high. If the dimension of $G . x$ is finite, then $N \in \mathcal{N}$ may be chosen in such a way that $\operatorname{dim} G / G_{x} N=\operatorname{dim} G . x$.

Note the relation between this result and Theorem 2.8 about neighboring orbits of a proper action on an arbitrary topological space. If that space is completely regular, then the conclusions of Theorem 2.8 are consequences of Theorem 3.8

Proof. Let $U \subseteq G$ be a compact neighborhood of $G_{x}$. When we apply Lemma 3.5 to $G / G^{1}$, we find an open subgroup $G_{0} \leq G$ such that $G_{x} \subseteq G_{0} \subseteq U G^{1}$. Since $U G^{1} / G^{1}$ is compact, so is $G_{0} / G^{1}$. Let $\mathcal{N}$ be the set of compact normal subgroups of $G_{0}$ such that $G_{0} / N$ is a Lie group. Then every identity neighborhood of $G$ contains a member of $\mathcal{N}$, and $\mathcal{N}$ is closed under finite intersections (Gluškov [24] $1.5]$ ), so that $\mathcal{N}$ is a filter basis that converges to 1 in $G$. Choose $N \in \mathcal{N}$. By Proposition 3.7, there is a $G_{x} N$-slice $S$ in $X$ for $G_{0}$ with $x \in S$. Lemma 3.6 shows that $S$ contains a $G_{x} N$-slice for the action of $G$ on $X$ that contains $x$. The coset space $G / G_{x} N$ is a manifold since it contains $G_{0} / G_{x} N$ as an open subspace and is paracompact (see, e.g., Bourbaki [10, Ch. III, §4, Prop. 13]). Since $\operatorname{dim} G . x=$ $\operatorname{dim} G_{0} / G_{x} \geq \operatorname{dim} G_{0} / G_{x} N=\operatorname{dim} G / G_{x} N$, the last assertions follow immediately from Lemma 3.4.

Remark 3.9. Let $G$ be a second countable locally compact group acting continuously on a metrizable space $X$. As a consequence of Theorem 3.8, if the action of $G$ on $X$ is proper and the orbit space is metrizable, then the action is proper in the sense of the Baum-Connes conjecture (see Baum, Connes, and Higson [3]). The reverse implication also holds. Details can be found in [7].

\section{ORBITS IN COHOMOLOGY MANIFOLDS}

The main result of this section (4.11) describes the orbits of highest dimension under a proper effective action on a cohomology manifold and gives an upper bound for the group dimension in terms of the orbit dimensions. If some orbit has codimension at most 2, then the group is a Lie group (4.13). This is prepared by the proof of continuity of induced actions in cohomology (4.2) and by a result on change of rings for cohomology manifolds (4.6).

We will use sheaf cohomology with compact supports and constant coefficients, which agrees with both Alexander-Spanier and Čech cohomology on locally compact spaces; see Bredon [16, III.2.1 and III.4.12].

Let $G$ be a connected group acting effectively on a Hausdorff space $X$, and let $N$ be a totally disconnected compact normal subgroup of $G$. Then there is an induced 
almost effective action of the quotient group $G / N$ on the orbit space $X / N$. The orbit projection $X \rightarrow X / N$ is a proper open map, and it induces a homeomorphism

$$
\operatorname{Fix}(G ; X) \approx \operatorname{Fix}\left(\frac{G}{N} ; \frac{X}{N}\right) .
$$

(Here $\operatorname{Fix}(G ; X)$ denotes the set of points in $X$ that are fixed under the action of $G$.) The orbit space of the action of $G / N$ on $X / N$ is homeomorphic to the orbit space $X / G$. If the action of $G$ on $X$ is proper, then the same holds for the action of $G / N$ on $X / N$.

If $G$ is a locally compact group of finite dimension such that $G / G^{1}$ is compact, then the totally disconnected compact normal subgroup $N$ may be chosen in such a way that $G / N$ is a Lie group. Thus, many questions about the orbit spaces and fixed point sets of actions of finite-dimensional locally compact groups are reduced to questions about Lie groups. This approach is very successful because the orbit space $X / N$ inherits global and local cohomological properties from the space $X$.

Theorem 4.1 (Bredon et al. 17, 5.1], Löwen [31). Let $N$ be a totally disconnected compact group that acts on a locally compact Hausdorff space $X$, and let $F$ be a field of characteristic 0 . Then the orbit projection $X \rightarrow X / N$ induces an isomorphism

$$
H_{c}^{*}(X / N ; F) \cong \operatorname{Fix}\left(N ; H_{c}^{*}(X ; F)\right) .
$$

If the dimension of the vector space $H_{c}^{*}(X ; F)$ is finite, the following theorem yields an open subgroup of $N$ that acts trivially on cohomology.

Theorem 4.2 (Continuity of the action on cohomology). Let $G$ be a locally paracompact Hausdorff group acting on a locally compact space $X$, and let $A$ be an abelian group. Then the induced action of $G$ on $H_{c}^{*}(X ; A)$ is continuous with respect to the discrete topology on $H_{c}^{*}(X ; A)$. (In other words, all stabilizers of this action are open subgroups.)

Proof. Choose a paracompact neighborhood $U$ of 1 in $G$. The proof will use the cohomology of $U \times X$ with constant coefficients in $A$ and with a special support family $\Phi$. This family is defined as follows. Let

$$
X \stackrel{\mathrm{pr}_{2}}{\longleftarrow} U \times X \stackrel{\omega}{\longrightarrow} X
$$

be the product projection and the restriction of the action map, respectively. The support family $\Phi$ is the collection of all closed subsets of sets of the form $\operatorname{pr}_{2}^{-1}(K) \cup$ $\omega^{-1}(K)$ where $K$ ranges over the compact subsets of $X$. Every closed subset of a member of $\Phi$ belongs to $\Phi$, and so does every finite union of members of $\Phi$. Thus $\Phi$ is indeed a family of supports in the sense of Bredon [16, I.6.1]. For a compact subset $K$ of $X$, the set $\operatorname{pr}_{2}^{-1}(K)=U \times K$ is paracompact (see Engelking [22] 5.1.36] or Bourbaki [10, Ch. I, $\S 9$, Prop. 17]). The set $\omega^{-1}(K)$ is the image of $U \times K$ under the self-homeomorphism $(g, x) \mapsto\left(g, g^{-1} . x\right)$ of $U \times X$, whence it is also paracompact. Finally, the union of the two paracompact sets $\operatorname{pr}_{2}^{-1}(K)$ and $\omega^{-1}(K)$ is paracompact (Engelking [22, 5.1.34]; cf. Dugundji [21, VIII.2.6]). This implies that every member of $\Phi$ is paracompact. Since $X$ is locally compact, every member of $\Phi$ has a neighborhood that belongs to $\Phi$. Thus $\Phi$ is a paracompactifying family of supports [16] I.6.1].

We will use a certain continuity property of the cohomology of subspaces of $U \times X$ with supports in $\Phi$. Let $\mathcal{N}$ be the collection of sets of the form $V \times X$ 
where $V$ is a closed neighborhood of 1 in $U$, and set $X_{1}:=\{1\} \times X=\bigcap \mathcal{N}$. For $N_{1}, N_{2} \in \mathcal{N}$ with $N_{1} \supseteq N_{2}$, we have the restriction map

$$
r_{N_{2}, N_{1}}^{*}: H_{\Phi \cap N_{1}}^{*}\left(N_{1} ; A\right) \longrightarrow H_{\Phi \cap N_{2}}^{*}\left(N_{2} ; A\right),\left.\quad \alpha \longmapsto \alpha\right|_{N_{2}},
$$

which is just the map in cohomology induced by the inclusion of $N_{2}$ into $N_{1}$. (Here $\Phi \cap N_{j}$ is the support family $\left\{F \cap N_{j} \mid F \in \Phi\right\}$ on $N_{j}$.) The family $\mathcal{N}$ is directed downwards by inclusion. Hence the groups $H_{\Phi \cap N}^{*}(N ; A)$, where $N$ ranges over $\mathcal{N}$, and the restriction maps form a directed system. In particular, the restriction maps $r_{X_{1}, N}^{*}$ induce a limit map

$$
\theta:=\lim _{\overrightarrow{N \in \mathcal{N}}} r_{X_{1}, N}^{*}: \lim _{N \in \mathcal{N}} H_{\Phi \cap N}^{*}(N ; A) \longrightarrow H_{\Phi \cap X_{1}}^{*}\left(X_{1} ; A\right) .
$$

(Note that $\Phi \cap X_{1}$ is just the family of compact supports on $X_{1}$.) The restriction of the product projection $\operatorname{pr}_{1}: U \times X \rightarrow U$ to an arbitrary element $F \in \Phi$ is a proper map. In particular, the image $\operatorname{pr}_{1}(F)$ is closed in $U$. Moreover, the topology of $U$ is regular. Hence if $F \in \Phi$ is disjoint from $X_{1}$, then there is an element $N \in \mathcal{N}$ such that $F \cap N=\emptyset$. Since $X_{1}$ and all members of $\mathcal{N}$ are closed subsets of $U \times X$ and $\Phi$ is a paracompactifying family of supports on $U \times X$, Bredon [16, II.10.6] proves that the direct limit map $\theta$ is an isomorphism. In particular, if two cohomology classes $\alpha, \alpha^{\prime} \in H_{\Phi}^{*}(U \times X ; A)$ satisfy $\left.\alpha\right|_{X_{1}}=\left.\alpha^{\prime}\right|_{X_{1}}$, then there is an element $N \in \mathcal{N}$ such that $\left.\alpha\right|_{N}=\left.\alpha^{\prime}\right|_{N}$.

Pick a cohomology class $\beta \in H_{c}^{*}(X ; A)$. We have to show that the stabilizer of $\beta$ in the induced action of $G$ on $H_{c}^{*}(X ; A)$ is open. Define elements of $H_{\Phi}^{*}(U \times X ; A)$ by $\alpha:=\operatorname{pr}_{2}{ }^{*}(\beta)$ and $\alpha^{\prime}:=\omega^{*}(\beta)$. (The maps $\operatorname{pr}_{2}{ }^{*}$ and $\omega^{*}$ are both defined since $\operatorname{pr}_{2}^{-1}(K), \omega^{-1}(K) \in \Phi$ for compact $K \subseteq X$.) We have $\left.\alpha\right|_{X_{1}}=\left.\alpha^{\prime}\right|_{X_{1}}$ because $\left.\operatorname{pr}_{2}\right|_{X_{1}}=\left.\omega\right|_{X_{1}}$. Therefore, there is a closed neighborhood $V$ of 1 in $U$ such that, for $N:=V \times X$, we have $\left.\alpha\right|_{N}=\left.\alpha^{\prime}\right|_{N}$. For $g \in U$, define an embedding

$$
i_{g}: X \longrightarrow U \times X, \quad x \longmapsto(g, x) .
$$

Then the action of the group element $g$ on $X$ is given by $\omega \circ i_{g}$, whence its action on $H_{c}^{*}(X ; A)$ is given by $i_{g}{ }^{*} \circ \omega^{*}$. Choose $g \in V$. Then $i_{g}$ factors as the corestriction $\left.i_{g}\right|^{N}: X \rightarrow N$ followed by the inclusion of $N$ into $U \times X$. Hence

$$
\begin{aligned}
i_{g}{ }^{*}\left(\omega^{*}(\beta)\right) & =i_{g}{ }^{*}\left(\alpha^{\prime}\right)=\left(\left.i_{g}\right|^{N}\right)^{*}\left(\left.\alpha^{\prime}\right|_{N}\right) \\
& =\left(\left.i_{g}\right|^{N}\right)^{*}\left(\left.\alpha\right|_{N}\right)=i_{g}{ }^{*}(\alpha)=\left(\operatorname{pr}_{2} \circ i_{g}\right)^{*}(\beta)=\left(\operatorname{id}_{X}\right)^{*}(\beta)=\beta .
\end{aligned}
$$

Therefore, the identity neighborhood $V \subseteq G$ fixes $\beta$ in the action of $G$ on $H_{c}^{*}(X ; A)$. Thus the stabilizer of $\beta$ is open.

Corollary 4.3. Let $G$ be a locally paracompact connected Hausdorff group acting on a locally compact space $X$, and let $A$ be an abelian group. Then the action of $G$ on $H_{c}^{*}(X ; A)$ is trivial.

For compact connected groups $G$, this corollary is due to Bredon, Raymond, and Williams [17, 2.2]. Bredon [16, II.11.11] gives a different proof of this special case, to which the present proof of Theorem 4.2 owes much inspiration.

In order to state a local analog of the global Theorem 4.1 we need the notion of a cohomology n-manifold over a principal ideal domain $R$. This is a locally compact space $X$ with $\operatorname{dim}_{R} X<\infty$ that is cohomologically locally connected in 
every degree and satisfies

$$
\mathcal{H}_{i}(X ; R)_{x} \cong \begin{cases}R & \text { if } i=n, \\ 0 & \text { if } i \neq n\end{cases}
$$

for all $x \in X$. Here $\mathcal{H}_{i}(X ; R)_{x}$ is the $i$-th local homology group of $X$ at $x$ with respect to Borel-Moore homology, the definition of which can be found in Bredon's monograph [16. Section V.3]. The cohomological local connectivity condition means that for each degree $i \in \mathbb{N}_{0}$ and for each point $x \in X$, every neighborhood $U$ of $x$ contains a neighborhood $V$ of $x$ such that the inclusion of $V$ into $U$ induces the zero map $\tilde{H}_{c}^{i}(U ; R) \rightarrow \tilde{H}_{c}^{i}(V ; R)$ in reduced cohomology. (In degree 0 , this is equivalent to ordinary local connectivity.) A connected cohomology $n$-manifold $X$ over $R$ is called orientable if $H_{c}^{n}(X ; R) \cong R$ (cf. [16. V.16.16]).

Equivalent definitions of a cohomology manifold are given by [16, V.16.8]. In particular, if $R$ is a field or the ring of integers, then the local homology groups may be replaced with the groups $H_{c}^{i}(X, X \backslash\{x\} ; R)$; see [16, II.12.1 and V.16.9]. Note that a cohomology $n$-manifold $X$ over $R$ satisfies $\operatorname{dim}_{R} X=n$ by [16, V.16.8].

Topological $n$-manifolds are examples of cohomology $n$-manifolds. A nonmanifold example is the open cone over an $(n-1)$-manifold which is not a sphere but has the $R$-cohomology of an $(n-1)$-sphere. Thus the open cone over a real projective space of odd dimension $n>1$ is a cohomology $(n+1)$-manifold over each field of characteristic different from 2 (but not over $\mathbb{Z}$ or over $\mathbb{Z} / 2$ ). Other non-manifold examples are provided by fixed point sets of elementary abelian or torus groups acting on manifolds and by cartesian factors of manifolds.

The main reference for cohomology manifolds is [16], in particular, Section V.16. An overview is given in Section 1.2 of [4]. The characterization of manifolds among cohomology manifolds is a hard open problem; see Bryant et al. [18].

As announced at the end of the introduction to Section 4 , the property of being a cohomology manifold over a field of characteristic 0 is inherited by certain orbit spaces.

Theorem 4.4 (Raymond [42]). Let $N$ be a second countable totally disconnected compact group that acts on a connected orientable cohomology $n$-manifold $X$ over some field $F$ of characteristic 0 . Suppose that the action of $N$ on $H_{c}^{n}(X ; F)$ is trivial. Then $X / N$ is an orientable cohomology n-manifold over $F$.

Remark 4.5. When we apply Raymond's Theorem 4.4 in the proof of Lemma 4.12 we will use Theorem 4.2 in order to choose the group $N$ in such a way that its action on $H_{c}^{n}(X ; F)$ will be trivial. The group will be second countable because it will be contained in a locally compact connected group of finite dimension (see Skljarenko [46, Theorem 18]).

We do not want to restrict our investigation to the case of orientable cohomology manifolds. If a cohomology manifold is not orientable, Bredon [12] has constructed an orientable covering (which may have infinitely many sheets), and under certain conditions, continuous actions on a non-orientable cohomology manifold are uniquely covered by orientation-preserving actions of the same group on the orientable covering ([12, 6.1]; cf. Bredon [14, I.9.4]). Unfortunately, these conditions are not satisfied in our situation, and a suitable more general development [13, Section III] was later found to be "irretrievably incorrect" (Bredon [16, V.9.6]). Our solution to the problem of non-orientability is to apply Raymond's Theorem 4.4 to 
orientable open subsets. Thus we can only derive "local" properties of an action from the corresponding properties of the action of a Lie group.

A different solution is available in the case of actions on compact cohomology manifolds. For this fact and further comments, see Remark 4.15 at the end of this paper.

Proposition 4.6 (Change of rings). Let $X$ be a cohomology n-manifold over some principal ideal domain $R$, and let $S$ be a principal ideal domain that is also a unital $R$-module. Then $X$ is a cohomology $n$-manifold over $S$. If $X$ is orientable over $R$, then $X$ is orientable over $S$.

Proof. For any locally compact space $U$, there is a natural exact Universal Coefficient Sequence (Bredon [16, II.15.3])

$$
0 \longrightarrow H_{c}^{i}(U ; R) \otimes_{R} S \longrightarrow H_{c}^{i}(U ; S) \longrightarrow \operatorname{Tor}_{1}^{R}\left(H_{c}^{i+1}(U ; R), S\right) \longrightarrow 0
$$

that splits. Applying this to open subsets $U \subseteq X$, we infer that $\operatorname{dim}_{S} X \leq$ $\operatorname{dim}_{R} X<\infty$. To see that $X$ is cohomologically locally connected over $S$, choose a degree $i \in \mathbb{N}_{0}$, a point $x \in X$, and a neighborhood $U$ of $x$. Then there are neighborhoods $V$ and $W$ of $x$ with $W \subseteq V \subseteq U$ such that the maps $H_{c}^{i+1}(V \hookrightarrow U ; R)$ and $H_{c}^{i}(W \hookrightarrow V ; R)$ are zero. We may assume that $V$ is open in $X$, so that it can take the place of $U$ in the above exact sequence. Then it follows from an elementary fact about short exact sequences [16. II.17.3] that the map $H_{c}^{i}(W \hookrightarrow U ; S)$ is zero. Finally, the local homology groups of $X$ over $S$ are determined by the exact sequence [16, V.3, (13)]

$$
0 \longrightarrow \mathcal{H}_{i}(X ; R)_{x} \otimes_{R} S \longrightarrow \mathcal{H}_{i}(X ; S)_{x} \longrightarrow \operatorname{Tor}_{1}^{R}\left(\mathcal{H}_{i-1}(X ; R)_{x}, S\right) \longrightarrow 0 .
$$

(Note that $\mathcal{H}_{i}(X ; S)_{x}$ does not depend on whether $S$ is considered as a module over $R$ or over $S$ because $X$ is cohomologically locally connected over $R$; see [16] V.12.10 and V.15.1].)

If $X$ is orientable over $R$, then the above Universal Coefficient Sequence in cohomology shows that $X$ is orientable over $S$.

Corollary 4.7. Every (orientable) cohomology n-manifold over $\mathbb{Z}$ is an (orientable) cohomology n-manifold over any principal ideal domain. Every (orientable) cohomology $n$-manifold over a principal ideal domain $R$ is an (orientable) cohomology $n$-manifold over the field of fractions of $R$.

The first assertion of this corollary is due to Borel et al. [9, I.4.5]. Conversely, let $X$ be a locally compact space that is cohomologically locally connected over $\mathbb{Z}$, and assume that $X$ is a (orientable) cohomology $n$-manifold over $\mathbb{Q}$ and over every prime field. Then $X$ is a (orientable) cohomology $n$-manifold over $\mathbb{Z}$. Together with the second assertion of the preceding corollary, this is a part of the main result of a paper of Raymond's 43 .

Consider the action of the circle group $\mathbb{T} \subseteq \mathbb{C}$ on the complex projective plane by $z \cdot\left[z_{1}, z_{2}, z_{3}\right]:=\left[z \cdot z_{1}, z_{2}, z_{3}\right]$. The fixed point set is the disjoint union of a point and a 2 -sphere. The next theorem shows that this situation is typical for torus actions.

Theorem 4.8 (Conner-Floyd). Let $R$ be a field or the ring of integers, and let the torus group $\mathbb{T}^{r}$ act on a connected cohomology $n$-manifold $X$ over $R$. Then $\operatorname{Fix}\left(\mathbb{T}^{r} ; X\right)$ is locally connected. Each connected component of $\operatorname{Fix}\left(\mathbb{T}^{r} ; X\right)$ is a cohomology $k$-manifold over $R$ for some $k$ such that $n-k$ is a nonnegative even 
number, and if $k=n$ (i.e., if the fixed point set has interior points), then the action is trivial. If $X$ is orientable over $R$, then so is each connected component of $\operatorname{Fix}\left(\mathbb{T}^{r} ; X\right)$.

This theorem also holds for actions of compact connected abelian groups on cohomology manifolds over fields of characteristic 0. Details of the proof, which follows the spirit of the present paper, and similar fixed point theorems for actions on cohomology spheres can be found in [6].

Proof. Except for the assertion about triviality of the action, this is Theorem V.3.2 of Borel et al. 9]. If $F$ is a connected component of $\operatorname{Fix}\left(\mathbb{T}^{r} ; X\right)$, then $F$ has interior points if and only if $\operatorname{dim}_{R} F=n$ (Bredon [16, V.16.18]). By Invariance of Domain [16, V.16.19], this holds if and only if $F$ is open in $X$, and this is equivalent to triviality of the action because $F$ is also closed and $X$ is connected.

We prepare our main result by a theorem that generalizes some of the main ideas of Bredon's paper 11] from actions of compact groups on manifolds to proper actions on cohomology manifolds. Bredon's use of local Lie groups is, at the same time, replaced with our Slice Theorem 3.8

As a comment on the hypotheses of the following theorem, we remark that for locally compact connected spaces, metrizability is equivalent to the second axiom of countability, i.e., the existence of a countable basis. Indeed, if a locally compact space is second countable, then Urysohn's Metrization Theorem applies (see Dugundji [21, IX.9.2]). Conversely, a metrizable space is paracompact. A locally compact connected space is paracompact if and only if it is $\sigma$-compact (see Bredon [15, I.12.11]). A $\sigma$-compact metric space is second countable because for each $n \in \mathbb{N}$, it can be covered by countably many open balls of radius $\frac{1}{n}$.

Theorem 4.9 (Orbits of codimension at most 2). Let a locally compact group $G$ act properly and effectively on a second countable connected cohomology n-manifold $X$ over some principal ideal domain $R$. Suppose that $\operatorname{dim} G . x \geq n-2$ holds for every orbit $G$. $x$ in $X$. Then $X$ is an $n$-manifold, and $G$ is a Lie group.

Proof. Let $x$ be a point of $X$. According to Theorem 3.8, we may choose a compact subgroup $N \leq G$ such that $G_{x}$ normalizes $N$, the coset space $G / G_{x} N$ is a manifold of dimension $\operatorname{dim} G . x$, and there is a $G_{x} N$-slice $S$ in $X$ for $G$ that contains $x$. Then the natural map of $G . S$ onto $G / G_{x} N$ is the projection in a locally trivial fiber bundle with fiber $S$. The open subset $G . S$ of $X$ is a cohomology $n$-manifold over $R$, and it is locally homeomorphic to $G / G_{x} N \times S$. A direct factor of a cohomology manifold is a cohomology manifold (see Bredon [16, V.16.11]), whence $S$ is a cohomology manifold of dimension $n-\operatorname{dim} G . x \leq 2$ over $R$. Since $S$ is second countable, we conclude that $S$ is a topological manifold ([16. V.16.32]; cf. [16, V.16.8]), whence the same holds for G.S. Since $x$ was an arbitrary point of $X$, the space $X$ is a manifold as well.

Let $S_{0}$ be the connected component of $x$ in $S$. An element $g \in N$ stabilizes $S_{0}$ as a set if and only if $g \cdot x \in S_{0}$. Since $S_{0}$ is open in $S$, the set-wise stabilizer $N_{S_{0}}$ is an open subgroup of $N$. The effective quotient $N_{S_{0}} / N_{\left[S_{0}\right]}$ embeds topologically into the set of self-homeomorphisms of $S_{0}$ with the compact-open topology (see Dugundji [21, XII.1.3 and 3.1]), and this space is second countable because so is $S_{0}$ (see [21, XII.5.2]). Hence $N_{S_{0}} / N_{\left[S_{0}\right]}$ is a second countable compact group that acts effectively on a connected manifold of dimension at most 2 . This situation has been 
studied by Montgomery and Zippin [38. If $\operatorname{dim}_{R} S=1$, then $N_{S_{0}} / N_{\left[S_{0}\right]}$ is a Lie group by Theorem 3 of Section 6.1 [38, p. 233]. If $\operatorname{dim}_{R} S=2$, then every compact zero-dimensional subgroup of $N_{S_{0}} / N_{\left[S_{0}\right]}$ is finite by the third theorem in Section 6.4 [38, p. 249]. Since the dimension of $N_{S_{0}} / N_{\left[S_{0}\right]}$ is finite (Montgomery [35]), this entails that $N_{S_{0}} / N_{\left[S_{0}\right]}$ is a Lie group; cf. Salzmann et al. [45, 96.31]. In particular, the orbit $N_{S_{0}} . x$ is a manifold, and so is $N . x$ because it contains $N_{S_{0}} . x$ as an open subset. By Proposition [3.2, the orbit G.x is a locally trivial fiber bundle over $G / G_{x} N$ with fiber $N . x$. We conclude that $G . x \approx G / G_{x}$ is a manifold. In particular, the space $G / G_{x}$ is locally connected for each $x \in X$. It follows that $G$ is a Lie group (see Bredon [11. Lemma 1]).

Remark 4.10. Let $G$ be a locally compact group acting properly on a second countable cohomology manifold $X$ over some principal ideal domain $R$, and let $Y \subseteq X$ be the set of points with orbits of codimension at most 2. Then $Y$ is an open subset of $X$ by Theorem 2.8 The preceding theorem shows that $Y$ is a manifold (or empty), and if $Y_{0}$ is a connected component of $Y$, then the set-wise stabilizer $G_{Y_{0}}$ is open in $G$ and the effective quotient $G_{Y_{0}} / G_{\left[Y_{0}\right]}$ is a Lie group.

In this situation, however, the space $X$ need not be locally Euclidean around points of the complement $X \backslash Y$. Indeed, let $I \cong A_{5}$ be an icosahedral subgroup of $\mathrm{SO}_{3} \mathbb{R}$. Then $\mathrm{SO}_{3} \mathbb{R} / I$ is a cohomology 3 -sphere that is not simply connected. Its suspension (i.e., double cone) is a compact cohomology 4 -manifold over $\mathbb{Z}$ that is not a manifold, and it carries an effective action of the group $\mathrm{SO}_{3} \mathbb{R} \times \mathbb{Z} / 2$.

Theorem 4.11 (Orbits of maximal dimension in cohomology manifolds). Let $G$ be a locally compact group acting properly and effectively on a connected cohomology $n$-manifold $X$ over $R$. Assume either that $R$ is a field of characteristic 0 or that $G$ is a Lie group and $R$ is a field or the ring of integers. Let $k$ be the highest occurring orbit dimension, and let $Y \subseteq X$ be the set of points with orbits of dimension $k$. Then the following statements hold:

(a) The subset $Y$ is open, connected, and dense in $X$, and its complement satisfies $\operatorname{dim}_{R}(X \backslash Y) \leq n-2$.

(b) For each $y \in Y$, the action of $G$ on the orbit $G . y$ is almost effective.

(c) $\operatorname{dim} G \leq\left(\begin{array}{c}k+1 \\ 2\end{array}\right)$.

(d) For each $y \in Y$, every orbit in $X$ meets $\operatorname{Fix}\left(\left(G_{y}\right)^{1} ; X\right)$. In other words,

$$
\forall x \in X, y \in Y \exists g \in G:\left(G_{y}\right)^{1} \subseteq G_{g \cdot x} .
$$

The theorem breaks down if $X$ is not connected. This is shown by suitable effective actions of (possibly infinite-dimensional) torus groups on disjoint unions of circles.

If $G$ is a compact Lie group and $R=\mathbb{Z}$, then the theorem follows from work by Yang [51] (cf. Borel et al. 9, Chapter IX]). In its present form, it rests on the following result, the proof of which partly follows a remark by Raymond [42, p. 6].

Lemma 4.12. Under the hypotheses of Theorem 4.11, if $G$ is connected and finitedimensional, then the boundary of $Y$ satisfies $\operatorname{dim}_{R} \partial Y \leq n-2$.

Proof. In the first part of the proof, assume that $G$ is a Lie group. Corollary 2.9 shows that $Y$ is an open subset of $X$. In particular, it is disjoint from its boundary $\partial Y$. For natural numbers $u$ and $v$, set

$$
B_{u, v}:=\left\{x \in \partial Y|\operatorname{dim} G . x=n-u,| G_{x} /\left(G_{x}\right)^{1} \mid=v\right\} .
$$


Our first aim is to show that $\operatorname{dim}_{R} B_{u, v} \leq n-2$. Choose $x \in B_{u, v}$. Palais's Slice Theorem [40, 2.3.3] yields a $G_{x}$-slice $S$ in $X$ that contains $x$. By Proposition 3.2. the space $G . S$ is a locally trivial fiber bundle over $G . x$ with fiber $S$, and $B_{u, v} \cap G . S$ is a locally trivial fiber bundle over $G . x$ with fiber $B_{u, v} \cap S$. In particular, we note that $S$ is a cohomology manifold of dimension $u$ over $R$ (see Bredon [16, V.16.11]). Hence the connected component of $x$ in $S$ is open in $S$, and it is invariant under $G_{x}$, so that we may suppose that $S$ is connected. If $x^{\prime} \in B_{u, v} \cap S$, then $G_{x^{\prime}} \subseteq G_{x}$ because $x^{\prime} \in S$, whence $G_{x^{\prime}}=G_{x}$ because $x^{\prime} \in B_{u, v}$. Since $G$.S meets $Y$, we may choose an element $y \in S \cap Y$. Then $G_{y}$ is a subgroup of $G_{x}$ of strictly smaller dimension. Therefore, there is a torus subgroup $T \leq G_{x}$ that is not contained in $G_{y}$. Hence the action of $T$ on $S$ is not trivial. Since $B_{u, v} \cap S \subseteq \operatorname{Fix}(T ; S)$, the Conner-Floyd Theorem 4.8 yields that $\operatorname{dim}_{R} B_{u, v} \cap S \leq u-2$, whence $\operatorname{dim}_{R} B_{u, v} \cap G . S \leq n-2$ by locality of dimension and the product inequality [16, II.16.8 and II.16.26]. We conclude from [16, II.16.8] that $\operatorname{dim}_{R} B_{u, v} \leq n-2$.

We will now prove that there is a pair $(u, v)$ such that $\operatorname{dim}_{R} \partial Y=\operatorname{dim}_{R} B_{u, v}$. Using the lexicographic ordering on $\mathbb{N} \times \mathbb{N}$, set

$$
B_{\leq(u, v)}:=\bigcup_{(s, t) \leq(u, v)} B_{s, t} \quad \text { and } \quad B_{<(u, v)}:=\bigcup_{(s, t)<(u, v)} B_{s, t} .
$$

Proposition 2.7 entails that each $B_{\leq(u, v)}$ and each $B_{<(u, v)}$ is an open subset of $\partial Y$. Since the dimension of $\partial Y$ is finite [16, II.16.8], there is a compact subset $K \subseteq \partial Y$ with $\operatorname{dim}_{R} K=\operatorname{dim}_{R} \partial Y$ (see [16, II.16.7]). The sets $B_{\leq(u, v)}$ form an ascending chain and cover $\partial Y$, whence one of them contains $K$ and therefore has the same dimension as $\partial Y$. Hence we may choose a lexicographically minimal pair $(u, v)$ such that $\operatorname{dim}_{R} B_{\leq(u, v)}=\operatorname{dim}_{R} \partial Y$. If $B_{<(u, v)}$ is empty, then $B_{\leq(u, v)}=B_{u, v}$ has the same dimension as $\partial Y$, and we are done. Otherwise, we may choose a compact subset $K^{\prime} \subseteq B_{<(u, v)}$ such that $\operatorname{dim}_{R} K^{\prime}=\operatorname{dim}_{R} B_{<(u, v)}$, and we find a pair $(s, t)<(u, v)$ such that $K^{\prime} \subseteq B_{\leq(s, t)}$ and hence

$$
\operatorname{dim}_{R} B_{<(u, v)}=\operatorname{dim}_{R} B_{\leq(s, t)}<\operatorname{dim}_{R} B_{\leq(u, v)},
$$

where the last inequality holds by the choice of $(u, v)$. Now $B_{\leq(u, v)}$ is the disjoint union of $B_{<(u, v)}$ and $B_{u, v}$, whence [16, p. 170, no. 11] yields that

$$
\operatorname{dim}_{R} \partial Y=\operatorname{dim}_{R} B_{\leq(u, v)}=\max \left\{\operatorname{dim}_{R} B_{<(u, v)}, \operatorname{dim}_{R} B_{u, v}\right\}=\operatorname{dim}_{R} B_{u, v} .
$$

We conclude that $\operatorname{dim}_{R} \partial Y \leq n-2$. Thus the lemma has been proved under the assumption that $G$ is a Lie group.

Now assume that $G$ is not a Lie group. Let $x_{0} \in \partial Y$ be an arbitrary point. We will construct a $G$-invariant connected open neighborhood $U$ of $x_{0}$ in $X$ and a totally disconnected compact normal subgroup $N$ of $G$ such that $G / N$ is a Lie group and the orbit space $U / N$ is a cohomology $n$-manifold over $R$. Then for any point $x$ of $U$, Skljarenko's sum formula for the dimension of coset spaces of locally compact groups [46. Theorem 10] entails that the dimension of the orbit of $N . x \in U / N$ under the action of $G / N$ on $U / N$ is equal to the dimension of G.x. Hence $(U \cap Y) / N$ is just the subset of $U / N$ formed by the orbits of dimension $k$. The boundary of this subset is $(U \cap \partial Y) / N$. We apply the first part of the proof to the action of the Lie group $G / N$ on the cohomology $n$-manifold $U / N$. This yields that $\operatorname{dim}_{R}(U \cap \partial Y) / N \leq n-2$. The orbit projection from $U \cap \partial Y$ onto $(U \cap \partial Y) / N$ is a continuous map between locally compact spaces and has totally disconnected fibers. By [16, IV.8.4], such a map cannot lower dimension. We conclude that 
$\operatorname{dim}_{R}(U \cap \partial Y) \leq n-2$. The lemma now follows from locality of dimension [16] II.16.8].

It remains to construct a $G$-invariant open neighborhood $U$ of $x_{0}$ in $X$ and a totally disconnected compact normal subgroup $N$ of $G$ with the required properties. The cohomology manifold $X$ is locally orientable [16, V.9.1 and V.16.8], which means that we may choose an orientable open neighborhood $V_{1}$ of $x_{0}$ in $X$. Since the action is continuous, we find an identity neighborhood $W$ in $G$ and a connected open neighborhood $V_{2}$ of $x_{0}$ in $X$ such that $W \cdot V_{2} \subseteq V_{1}$. Since $G$ can be approximated by Lie groups, the identity neighborhood $W$ contains a totally disconnected compact normal subgroup $N_{1}$ of $G$ such that $G / N_{1}$ is a Lie group and $N_{1} . x_{0} \subseteq V_{2}$. The latter property implies that the open subset $V_{3}:=N_{1} . V_{2}$ of $X$ is connected. Therefore, the $R$-module $H_{c}^{n}\left(V_{3} ; R\right)$ is isomorphic to $R$, and Theorem 4.2 shows that every sufficiently small subgroup of $N_{1}$ acts trivially on $H_{c}^{n}\left(V_{3} ; R\right)$. Hence $N_{1}$ contains a compact normal subgroup $N$ of $G$ such that $G / N$ is a Lie group and the action of $N$ on $H_{c}^{n}\left(V_{3} ; R\right)$ is trivial. Since $N$ is second countable (Skljarenko [46, Theorem 18]) and $R$ is now assumed to be a field of characteristic 0, Raymond's Theorem 4.4 shows that $V_{3} / N$ is a cohomology $n$-manifold over $R$. Set $U:=G \cdot V_{3}$. Then $U$ is a connected open neighborhood of $x_{0}$, and $U / N=G$. $\left(V_{3} / N\right)$ is a cohomology $n$-manifold over $R$. Thus $U$ and $N$ have been constructed as announced.

Proof of Theorem 4.11. Skljarenko's sum formula [46, Theorem 10] implies that $\operatorname{dim} G^{1} \cdot x=\operatorname{dim} G . x$ for every $x \in X$. Hence the theorem follows for the action of $G$ if it can be proved for the action of the identity component $G^{1}$. Therefore, we will assume that $G$ is connected. In the first part of the proof, we will also assume that the dimension of $G$ is finite, so that we can apply Lemma 4.12

Corollary 2.9 yields that $Y$ is open in $X$. Therefore, the complement of $\partial Y$ in $X$ is the disjoint union of the open sets $Y$ and $X \backslash \bar{Y}$. Since $\operatorname{dim}_{R} \partial Y \leq n-2$, the complement $X \backslash \partial Y$ is connected (Bredon [16, V.16.20]). Hence $X \backslash \bar{Y}$ is empty. Thus $Y$ is dense in $X$ and connected, and the dimension of $X \backslash Y=\partial Y$ over $R$ is at most $n-2$. This proves (a).

The action of $G$ on the dense subset $Y$ of $X$ is effective. Therefore, assertions (b) and (c) follow from Corollary 2.10, which also shows that the identity components of stabilizers of points in $Y$ form a single conjugacy class. Let $x \in X$ be an arbitrary point. Using again that $Y$ is a dense subset of $X$, we infer from Theorem 2.8 that $G_{x}$ has a subgroup that is conjugate to $\left(G_{y}\right)^{1}$ for some $y \in Y$. In other words, there is an element $g \in G$ such that $\left(G_{y}\right)^{1} \subseteq g G_{x} g^{-1}=G_{g . x}$. Moreover, we have seen that this can be achieved for an arbitrary point $y$ of $Y$, which proves (d).

To complete the proof of Theorem 4.11, assume that $G$ is a connected group of infinite dimension. We have already seen that any closed finite-dimensional subgroup of $G$ has dimension at most $\left(\begin{array}{c}k+1 \\ 2\end{array}\right)$. The Mal'cev-Iwasawa Theorem (see Hofmann and Terp 27) yields a maximal compact subgroup $K$ of $G$ that is connected and has infinite dimension. The structure theory of compact groups shows that there is a totally disconnected normal subgroup $N$ of $K$ such that $K / N$ is isomorphic to the product over a (necessarily infinite) family of compact connected Lie groups (see Hofmann and Morris [26] 8.15 and 9.24]). We conclude that $K$ contains closed subgroups of arbitrarily high dimension, which is a contradiction. 
Corollary 4.13. Let $G$ be a locally compact group acting properly and effectively on a second countable connected cohomology manifold $X$ over a field of characteristic 0 . If some orbit has codimension at most 2 , then $G$ is a Lie group.

Note that a one-dimensional solenoid can act effectively on the cartesian product $\mathbb{S}^{1} \times \mathbb{N}$.

For a compact group acting on a second countable connected cohomology manifold over $\mathbb{Z}$ of finite covering dimension, the corollary is due to Raymond [42].

Proof. Theorem 4.11 yields that the action of $G$ on the connected subset $Y \subseteq X$ formed by the orbits of minimal codimension is effective. Therefore, the corollary follows from Theorem 4.9

Corollary 4.14. Every locally compact group that can act effectively on a connected cohomology manifold over a field of characteristic 0 has finite dimension.

For actions on genuine manifolds, this is due to Montgomery [35].

Proof. By Theorem 4.11, every compact subgroup has finite dimension, whence the corollary follows from the Mal'cev-Iwasawa Theorem (see Hofmann and Terp [27; cf. Abels [1]), applied to the identity component.

Remark 4.15. Let $G$ be a locally compact connected group that acts effectively on a connected cohomology $n$-manifold $X$ over a field $F$ of characteristic 0 . Then the dimension of $G$ is finite. If $X$ is orientable, then for any totally disconnected compact subgroup $N$ of $G$, the orbit space $X / N$ is a cohomology $n$-manifold over $F$. (This follows from Raymond's Theorem 4.4 the hypotheses of which are satisfied by Corollary 4.3 and Skljarenko [46, Theorem 18].) The subgroup $N$ may be chosen to be normal and such that the quotient $G / N$ is a Lie group. Thus, the action of $G$ on $X$ is closely related to the action of the Lie group $G / N$ on the cohomology $n$-manifold $X / N$.

If $X$ is not assumed to be orientable, we have the following partial substitute for this technique. Let $K$ be a compact subset of $X$. Then there are open connected orientable subsets $U_{1}, \ldots, U_{m}$ of $X$ and totally disconnected compact normal subgroups $N_{1}, \ldots, N_{m}$ of $G$ such that the sets $U_{j}$ cover $K$, the quotient groups $G / N_{j}$ are Lie groups, each set $U_{j}$ is invariant under the group $N_{j}$, and the action of $N_{j}$ on $H_{c}^{n}\left(U_{j} ; F\right)$ is trivial. (This can be deduced from Theorem 4.2 as in the proof of Lemma 4.12) Set $U:=U_{1} \cup \cdots \cup U_{m}$ and $N:=N_{1} \cap \cdots \cap N_{m}$. Then $U$ is an open subset of $X$ that contains $K$, and if $K$ is connected, then so is $U$. Gluškov 24. $1.5]$ has proved that the quotient $G / N$ is a Lie group. By Raymond's Theorem 4.4 each orbit space $U_{j} / N$ is a cohomology $n$-manifold over $F$. We conclude that the open subspace $G . U / N$ of $X / N$, which contains $G . K / N$ and is connected if $K$ is connected, is a cohomology $n$-manifold over $F$, and it carries an action of the Lie group $G / N$.

In particular, if the cohomology manifold $X$ is compact, then the group $G$ has a totally disconnected compact normal subgroup $N$ such that $G / N$ is a Lie group and $X / N$ is a cohomology $n$-manifold over $F$ (even if $X$ is not orientable).

\section{REFERENCES}

[1] Herbert Abels, Parallelizability of proper actions, global K-slices and maximal compact subgroups, Math. Ann. 212 (1974/75), 1-19. MR 51:11460 
[2] Christopher Allday and Volker Puppe, Cohomological methods in transformation groups, Cambridge Studies in Advanced Mathematics 32, Cambridge University Press, 1993. MR 94g:55009

[3] Paul Baum, Alain Connes, and Nigel Higson, Classifying space for proper actions and $K$ theory of group $C^{*}$-algebras, $C^{*}$-algebras: 1943-1993 (San Antonio, TX, 1993), Amer. Math. Soc., Providence, RI, 1994, pp. 240-291. MR 96c:46070

[4] Harald Biller, Actions of compact groups on spheres and on generalized quadrangles, Dissertation, Stuttgart, 1999, http://elib.uni-stuttgart.de/opus/volltexte/1999/566.

[5] — Actions of compact groups on compact $(4, m)$-quadrangles, Geom. Dedicata 83 (2000), 273-312. MR 2001m:51021

[6] _ Fixed points of pro-tori in cohomology spheres, Preprint 2183, Fachbereich Mathematik, Technische Universität Darmstadt, 2001, http://wwwbib.mathematik.tu-darmstadt. de/Math-Net/Preprints/Listen/shadow/pp2183.html.

[7] _ Characterizations of proper actions, Preprint 2211, Fachbereich Mathematik, Technische Universität Darmstadt, 2002, http://wwwbib.mathematik.tu-darmstadt.de/ Math-Net/Preprints/Listen/shadow/pp2211.html; to appear in Math. Proc. Cambridge Philosophical Society.

[8] Salomon Bochner and Deane Montgomery, Locally compact groups of differentiable transformations, Ann. of Math., II. Ser. 47 (1946), 639-653. MR 8:253c

[9] Armand Borel et al., Seminar on transformation groups, Ann. of Math. Stud. 46, Princeton University Press, Princeton, 1960. MR 22:7129

[10] Nicolas Bourbaki, Éléments de mathématique. Topologie générale. Chapitres 1 à 4, Hermann, Paris, 1971 (French). MR 50:11111

[11] Glen E. Bredon, Some theorems on transformation groups, Ann. of Math., II. Ser. 67 (1958), 104-118. MR 20:83

[12] - Orientation in generalized manifolds and applications to the theory of transformation groups, Michigan Math. J. 7 (1960), 35-64. MR 22:7130

[13] Cohomology fibre spaces, the Smith-Gysin sequence, and orientation in generalized manifolds, Michigan Math. J. 10 (1963), 321-333. MR 28:2546

[14] _ Introduction to compact transformation groups, Academic Press, New York, 1972. MR 54:1265

[15] — Topology and geometry, Graduate Texts in Mathematics 139, Springer, New York, 1993. MR 94d:55001

[16] _ Sheaf theory, 2nd ed., Graduate Texts in Mathematics 170, Springer, New York, 1997. MR 98g:55005

[17] Glen E. Bredon, Frank Raymond, and R. F. Williams, p-Adic groups of transformations, Trans. Amer. Math. Soc. 99 (1961), 488-498. MR 26:251

[18] John L. Bryant, Steven C. Ferry, Washington Mio, and Shmuel Weinberger, Topology of homology manifolds, Ann. of Math., II. Ser. 143 (1996), 435-467. MR 97b:57017

[19] Francis Buekenhout (ed.), Handbook of incidence geometry: Buildings and foundations, North-Holland, Amsterdam, 1995. MR 96e:51002

[20] Tammo tom Dieck, Transformation groups, Studies in Mathematics 8, de Gruyter, Berlin, 1987. MR 89c:57048

[21] James Dugundji, Topology, Allyn and Bacon, Boston, 1966. MR 33:1824

[22] Ryszard Engelking, General topology, 2nd ed., Sigma Series in Pure Mathematics 6, Heldermann-Verlag, Berlin, 1989. MR 91c:54001

[23] Ryszard Engelking, Dimension theory, North-Holland Mathematical Library 19, NorthHolland, New York, 1978. MR 58:2753b

[24] Viktor M. Gluškov, The structure of locally compact groups and Hilbert's fifth problem, Amer. Math. Soc. Transl., II. Ser. 15 (1960), 55-93, transl. of Uspekhi Mat. Nauk. 12 (1957), no. 2, 3-41. MR 22:5690, MR 21:698

[25] Karl H. Hofmann and Sidney A. Morris, Transitive actions of compact groups and topological dimension, J. Algebra 234 (2000), 454-479. MR 2002b:22006

[26] _ The structure of compact groups, Studies in Mathematics 25, de Gruyter, Berlin, 1998. MR 99k:22001

[27] Karl H. Hofmann and Christian Terp, Compact subgroups of Lie groups and locally compact groups, Proc. Amer. Math. Soc. 120 (1994), no. 2, 623-634. MR 94d:22003 
[28] Wu-Yi Hsiang, Cohomology theory of topological transformation groups, Ergebnisse der Mathematik und ihrer Grenzgebiete 85, Springer, Berlin, 1975. MR 54:11363

[29] Irving Kaplansky, Lie algebras and locally compact groups, Chicago Lectures in Mathematics, The University of Chicago Press, 1971. MR 43:2145

[30] Linus Kramer, Compact polygons, Dissertation, Tübingen, 1994.

[31] Rainer Löwen, Locally compact connected groups acting on Euclidean space with Lie isotropy groups are Lie, Geom. Dedicata 5 (1976), 171-174. MR 54:7691

[32] _ Topology and dimension of stable planes: On a conjecture of H. Freudenthal, J. Reine Angew. Math. 343 (1983), 108-122. MR 85b:57020

[33] Gaven J. Martin, The Hilbert-Smith conjecture for quasiconformal actions, Electron. Res. Announc. Amer. Math. Soc. 5 (1999), 66-70 (electronic). MR 2000c:30044

[34] Deane Montgomery, Topological groups of differentiable transformations, Ann. of Math., II. Ser. 46 (1945), 382-387. MR 7:114h

[35] _ Finite dimensionality of certain transformation groups, Illinois J. Math. 1 (1957), 28-35. MR 18:745c

[36] Deane Montgomery and Leo Zippin, Topological transformation groups I, Ann. of. Math., II. Ser. 41 (1940), 778-791.

[37] — A theorem on Lie groups, Bull. Amer. Math. Soc. 48 (1942), 448-452. MR 4:3a

[38] - Topological transformation groups, Interscience, New York, 1955. MR 17:383b

[39] Paul S. Mostert, Sections in principal fibre spaces, Duke Math. J. 23 (1956), 57-71. MR 17:771f

[40] Richard S. Palais, On the existence of slices for actions of non-compact Lie groups, Ann. of Math., II. Ser. 73 (1961), 295-323. MR 23:A3802

[41] Boris A. Pasynkov, On the coincidence of various definitions of dimensionality for factor spaces of locally bicompact groups, Uspekhi Mat. Nauk. 17 (1962), no. 5, 129-135 (Russian). MR 26:2538

[42] Frank Raymond, The orbit spaces of totally disconnected groups of transformations on manifolds, Proc. Amer. Math. Soc. 12 (1961), 1-7. MR 25:5127

[43] - Some remarks on the coefficients used in the theory of homology manifolds, Pacific J. Math. 15 (1965), 1365-1376. MR 32:6448

[44] Dušan Repovš and Evgenij V. Ščepin, A proof of the Hilbert-Smith conjecture for actions by Lipschitz maps, Math. Ann. 308 (1997), no. 2, 361-364. MR 99c:57066

[45] Helmut Salzmann, Dieter Betten, Theo Grundhöfer, Hermann Hähl, Rainer Löwen, and Markus Stroppel, Compact projective planes, de Gruyter, Berlin, 1995. MR 97b:51009

[46] E. G. Skljarenko, On the topological structure of locally bicompact groups and their quotient spaces, Amer. Math. Soc. Transl., II. Ser. 39 (1964), 57-82, transl. of Mat. Sbornik (N.S.) 60 (1963), 63-88. MR 26:5090

[47] Bernhild Stroppel and Markus Stroppel, The automorphism group of a compact generalized quadrangle has finite dimension, Arch. Math. 66 (1996), 77-79. MR 96i:51014

[48] _ The automorphism group of a compact generalized polygon has finite dimension, Monatsh. Math. 127 (1999), 343-347. MR 2000f:51025

[49] Harald Upmeier, Symmetric Banach manifolds and Jordan $C^{*}$-algebras, Mathematics Studies 104, North-Holland Publishing Co., Amsterdam, 1985. MR 87a:58022

[50] Hidehiko Yamabe, A generalization of a theorem of Gleason, Ann. of Math., II. Ser. 58 (1953), 351-365. MR 15:398d

[51] Chung-Tao Yang, Transformation groups on a homological manifold, Trans. Amer. Math. Soc. 87 (1958), 261-283. MR 20:6704

[52] _

Fachbereich Mathematik, Technische Universität Darmstadt, SchlossgartenStrasse 7, 64289 Darmstadt, Germany

E-mail address: biller@mathematik.tu-darmstadt.de 